\title{
Chemical equilibration of quarks and gluons at RHIC and LHC energies
}

\author{
Duncan M. Elliott ${ }^{1}$ and Dirk H. Rischke ${ }^{2}$ \\ ${ }^{1}$ Department of Physics \\ University of Cape Town, Rondebosch 7701, South Africa \\ email: elliott@physci.uct.ac.za \\ ${ }^{2}$ RIKEN-BNL Research Center \\ Brookhaven National Laboratory, Upton, New York 11973, U.S.A. \\ email: rischke@bnl.gov
}

\begin{abstract}
We study chemical equilibration of quarks and gluons in central nuclear collisions at RHIC and LHC energies. The initial quark and gluon densities are taken from earlier studies as well as from recent perturbative QCD estimates and are then evolved via rate equations coupled to longitudinally boost-invariant fluid dynamics. We find that, for RHIC initial conditions, the lifetime of quark-gluon matter is too short in order for the quark and gluon number densities to chemically equilibrate prior to hadronization. In contrast, at LHC energies chemical equilibration is complete before the system hadronizes. Entropy production due to chemical equilibration can be as large as $30 \%$.
\end{abstract}

\section{Introduction}

Nucleus-nucleus collisions at ultrarelativistic energies probe the properties of nuclear matter under extreme conditions [1]. Of particular interest is the question whether ordinary nuclear matter undergoes a (phase) transition to quark-gluon matter, as predicted by lattice calculations of quantum chromodynamics (QCD) [2].

A nuclear collision can be viewed as a sequence of nucleon-nucleon collisions. At sufficiently high energies, multi-particle production leads to the formation of a region of high energy and particle number density. With increasing beam energy, multi-particle production through processes where the partons inside the nucleons directly interact with each other, becomes more and more important. The dominant partonic particle-production mechanism is so-called mini-jet production [3].

If the momentum transfer in these interactions is sufficiently large, mini-jet production is

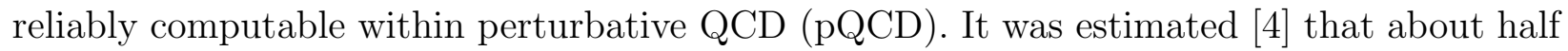
of the total transverse energy created in $A u+A u$ collisions at the Relativistic Heavy-Ion 
Collider (RHIC) at BNL resides in mini-jets, while at CERN's Large Hadron Collider (LHC) the transverse energy created is almost exclusively due to mini-jets.

The transverse energy can be used to estimate the energy densities created in the initial stage of the collision. If matter is in thermodynamical equilibrium, both for RHIC and LHC energies the corresponding energy densities are found to be large enough for nuclear matter to be in the quark-gluon phase. The remaining question is whether high-energy density matter formed in ultrarelativistic nuclear collisions lives sufficiently long enough to actually reach thermodynamical equilibrium, so that it can be identified with the quark-gluon phase seen in lattice QCD calculations.

Thermodynamical equilibrium means that matter is in thermal, mechanical, and chemical equilibrium. In general, the question of thermodynamical equilibration can only be decided with microscopic transport models [5]. In this paper, we study a simpler problem which allows us to use a macroscopic transport model. We assume that matter reaches thermal and mechanical equilibrium after a proper time $\tau_{0} \sim 0.1-0.25 \mathrm{fm} / \mathrm{c}$. This is justified given the fact that the rate for elastic collisions between quarks and gluons, which establish thermal and mechanical equilibrium, is much larger than for inelastic collisions which establish chemical equilibrium. Thermal and mechanical equilibrium will be referred to as kinetic equilibrium in the following.

Under these assumptions, and given initial values for the energy density and the quark, antiquark and gluon number densities, we can then employ ideal fluid dynamics to study the subsequent evolution of the kinetically equilibrated quark-gluon phase, coupled to rate equations which determine the chemical composition of the system away from chemical equilibrium. This problem has been previously studied by Biró et al. [6], and by Srivastava et al. [7, 8]. As in these previous studies, we assume that chemical equilibration is driven mainly by the two-body reaction $g g \leftrightarrow q \bar{q}$, and by gluon multiplication as well as fusion, $g g \leftrightarrow g g g$. We also terminate the evolution when the plasma reaches the hadronization energy density, $\epsilon_{h} \equiv 1.45 \mathrm{GeV} / \mathrm{fm}^{3}$. For a completely equilibrated plasma, this corresponds to a hadronization temperature of $T_{h} \sim 0.17 \mathrm{GeV}$. We do not study the evolution in the mixed and purely hadronic phases.

Our motivation to reinvestigate this subject is the following. First of all, the question whether the quark-gluon phase created in ultrarelativistic heavy-ion collisions is chemically equilibrated is highly relevant for the experiments commencing at the RHIC collider in the fall of this year. Since [6, 7, 8] were published, newer results on mini-jet production in nuclear collisions at RHIC and LHC energies have been obtained [⿴囗十 to compare the evolution computed with initial conditions obtained from the so-called "selfscreened parton cascade" (SSPC) model (used in [7, 8]) with that employing the more recent pQCD estimates of [4].

Second, the authors of [6, 7, 8] simplified the rate equations using an approximate, socalled "factorized" phase-space distribution function for the quarks and gluons (see below). In our treatment we use the full distribution function in the rate equations, and so are in a position to assess the validity of the factorization assumption.

Third, baryon stopping is non-negligible in nuclear collisions at SPS energies [9], and there is mounting evidence from theoretical studies that, even at RHIC energies, the midrapidity region is not completely net-baryon free [10]. Our analysis therefore also accounts for nonzero net-baryon number. 
Finally, the numerical algorithms used here are different from the ones employed before [7, 8. Thus, they constitute an independent check on the validity of the conclusions reached previously.

This paper is organized as follows. In section 2 we discuss the macroscopic transport equations. Starting from a single-particle phase-space distribution in kinetic equilibrium, we show that these equations are given by ideal fluid dynamics, coupled to rate equations which determine the densities of the individual particle species. We also prove that the entropy never decreases during the time evolution of the system. In section 3 we discuss the initial conditions for the transport equations. Section 4 is devoted to purely longitudinal boost-invariant expansion. We show that the difference in the equilibration process is small when using the full phase-space distribution function instead of the factorized distribution function of [6, 7, 8]. We compare results obtained with the initial conditions of the SSPC model [7, 8] with those when using pQCD estimates [4]. We also study the sensitivity of the equilibration process on the initial time, $\tau_{0}$, and the strong coupling constant, $\alpha_{s}$. In section 5 we include (cylindrically symmetric) transverse expansion as well, and present results for the SSPC model. We conclude in section 6 with a summary of our results and an outlook. Our units are $\hbar=c=k_{B}=1$, and the metric tensor is $g^{\mu \nu}=\operatorname{diag}(+,-,-,-)$.

\section{Macroscopic transport equations}

In this section, we discuss the macroscopic transport equations on which our results are based. We assume that elastic collisions between quarks (antiquarks) and gluons are sufficiently frequent to establish kinetic equilibrium. Ideal fluid dynamics can then be invoked to follow the evolution of the energy and momentum densities in the system, while rate equations for the inelastic reactions $g g \leftrightarrow g g g$ and $g g \leftrightarrow q \bar{q}$ determine the number density of quarks, antiquarks, and gluons.

\subsection{Phase-space distribution}

Once kinetic equilibrium is achieved, all particles in an (infinitesimal) volume element at space-time point $x \equiv x^{\mu}=(t, \mathbf{x})$ have the same temperature, $T(x)$, and move with a common average 4-velocity, $u(x) \equiv u^{\mu}(x)$. In that case, the single-particle phase-space distribution for particles of species $i$ assumes the same form as in (local) thermodynamical equilibrium, except that the fugacity (or the chemical potential) is not equal to its equilibrium value:

$$
f_{i}(x, k \cdot u)=\lambda_{i}(x) \frac{d_{i}}{(2 \pi)^{3}} \frac{1}{\exp [k \cdot u(x) / T(x)]+\lambda_{i}(x) \theta_{i}},
$$

where $k \equiv k^{\mu}=\left(E_{i}, \mathbf{k}\right)$ is the 4-momentum; $E_{i}=\sqrt{\mathbf{k}^{2}+m_{i}^{2}}$ is the on-shell energy of particles of species $i$ with 3 -momentum $\mathbf{k}$ and rest mass $m_{i}$. The fugacity $\lambda_{i}(x)=\exp \left[\mu_{i}(x) / T(x)\right]$ controls the number density of particle species $i, \mu_{i}$ is the chemical potential. $\theta_{i}= \pm 1$ for fermions or bosons, respectively. $d_{i}$ denotes the number of internal degrees of freedom for particles of species $i$ (like spin, isospin, color, etc.). 
In [6, 0, 8], instead of (11) a so-called factorized distribution function was used, in which the fugacity $\lambda_{i}$ in the denominator is approximated by 1 ,

$$
f_{i}^{\mathrm{fac}}(x, k \cdot u) \equiv \lambda_{i}(x) \frac{d_{i}}{(2 \pi)^{3}} \frac{1}{\exp [k \cdot u(x) / T(x)]+\theta_{i}} .
$$

We will comment on the validity of this approximation below.

\subsection{Energy-momentum conservation}

With a distribution function of the type (11), the energy-momentum tensor assumes the so-called ideal-fluid form

$$
T^{\mu \nu}(x) \equiv \sum_{i} \int \frac{\mathrm{d}^{3} \mathbf{k}}{E_{i}} k^{\mu} k^{\nu} f_{i}(x, k \cdot u)=[\epsilon(x)+p(x)] u^{\mu}(x) u^{\nu}(x)-p(x) g^{\mu \nu}
$$

where

$$
\epsilon(x) \equiv \sum_{i} \int \mathrm{d}^{3} \mathbf{k} E_{i} f_{i}\left(x, E_{i}\right), \quad p(x) \equiv \sum_{i} \int \mathrm{d}^{3} \mathbf{k} \frac{\mathbf{k}^{2}}{3 E_{i}} f_{i}\left(x, E_{i}\right)
$$

are the energy density and pressure in the local rest frame of a fluid element moving with 4-velocity $u^{\mu}$.

While it is clear that thermal equilibrium requires all particle species to have the same temperature, it is less obvious why the phase-space distribution (11) ensures mechanical equilibrium as well. To see this, consider the tensor decomposition $T^{\mu \nu}$ as given by (3). In the rest frame of the fluid element, the pressure is completely isotropic, $p \equiv T^{i i} / 3=T^{x x}=$ $T^{y y}=T^{z z}$, which is synonymous to mechanical equilibrium. If (1) depends on more than one 4-vector $u$, additional tensors would appear on the right-hand side of (3), such as $u_{k}^{\mu} u_{l}^{\nu}$, and the diagonal components of the stress tensor $T^{i j}$ would no longer be identical. Hence, the common 4 -velocity $u$ in (11) ensures mechanical equilibrium.

In our case, matter consists of massless gluons, quarks, and antiquarks, $i=g, q$, $\bar{q}$, with energy density

$$
\begin{aligned}
\epsilon & =\epsilon_{g}+\epsilon_{q}+\epsilon_{\bar{q}} \\
\epsilon_{g} & =\lambda_{g} T^{4} \frac{d_{g}}{2 \pi^{2}} \int_{0}^{\infty} \mathrm{d} z \frac{z^{3}}{e^{z}-\lambda_{g}}, \\
\epsilon_{q} & =\lambda_{q} T^{4} \frac{d_{q}}{2 \pi^{2}} \int_{0}^{\infty} \mathrm{d} z \frac{z^{3}}{e^{z}+\lambda_{q}}, \\
\epsilon_{\bar{q}} & =\lambda_{\bar{q}} T^{4} \frac{d_{q}}{2 \pi^{2}} \int_{0}^{\infty} \mathrm{d} z \frac{z^{3}}{e^{z}+\lambda_{\bar{q}}},
\end{aligned}
$$

where $d_{g} \equiv 2\left(N_{c}^{2}-1\right)$ is the number of internal degrees of freedom for gluons, $N_{c}=3$ is the number of colors, and $d_{q} \equiv 2 N_{c} N_{f}$, is the number of internal degrees of freedom for quarks and antiquarks, with $N_{f}$ being the number of massless flavors. Throughout our analysis, we use $N_{f}=2.5$, mimicking the effect of the nonzero mass of the strange quark by taking $d_{s}=0.5 d_{q}$. 
This approximation interpolates between the region of high temperature, $T \gg m_{s} \simeq 150$ $\mathrm{MeV}$, where $N_{f} \simeq 3$, and the region of low temperature, $m_{u, d} \ll T \ll m_{s}$, where $N_{f} \simeq 2$. Since we follow the time evolution of the temperature from very high $T$ down to $T \simeq T_{c} \sim m_{s}$, taking $N_{f}=2.5$ is not a particularly good approximation. We nevertheless make this choice to be able to compare our results to earlier work [6, 7, 8]. Also, the chemical reaction rates, cf. eqs. (22) below, which appear on the right-hand side of the rate equations (19) - (21), are more complicated in the case of massive particles [11]. The extension of our present study to nonzero strange quark mass is, however, important and will be pursued in a subsequent publication 12 .

For massless bosons, the equilibrium value of the fugacity, $\lambda_{i}^{\mathrm{eq}}$, is always equal to 1 . The reason is the following. For bosons, the equilibrium value of the chemical potential, $\mu_{i}^{\text {eq }}$, has to be smaller than the rest mass, $\mu_{i}^{\text {eq }} \leq m_{i}$. On the other hand, bosons which carry a conserved charge, $\mu_{i}^{\text {eq }} \neq 0$, always come in pairs with their own antiparticles, e.g., $\pi^{+}$and $\pi^{-}, K$ and $\bar{K}$, etc. Without loss of generality, we can therefore choose $\mu_{i}^{\text {eq }} \geq 0$ (with the chemical potential of the associated antiparticle $\left.\mu_{\bar{i}}^{\mathrm{eq}}=-\mu_{i}^{\mathrm{eq}} \leq 0\right)$. Then, for massless bosons, $\mu_{i}^{\mathrm{eq}} \equiv 0$, such that $\lambda_{i}^{\mathrm{eq}}=1$.

For massless fermions, there is no such constraint, although $\lambda_{q}^{\mathrm{eq}} \equiv 1 / \lambda_{\bar{q}}^{\mathrm{eq}}$ in all cases and $\lambda_{q}^{\mathrm{eq}} \equiv \lambda_{\bar{q}}^{\mathrm{eq}}=1$ for vanishing net-baryon density.

The pressure is related to the energy density through

$$
p \equiv \frac{\epsilon}{3},
$$

the well-known equation of state for an ultrarelativistic ideal gas. Note that, in order to derive this relation, we only required the system to be in kinetic equilibrium, i.e., eq. (9) is valid even when the system is not in chemical equilibrium!

With the factorized distribution function (2), the integrals in (6) - (8) can be performed analytically, as they no longer depend on the fugacities. The energy densities simplify to

$$
\epsilon_{g}^{\mathrm{fac}}=a_{2} \lambda_{g} T^{4} \quad, \quad \epsilon_{q}^{\mathrm{fac}}=b_{2} \lambda_{q} T^{4} \quad, \quad \epsilon_{\bar{q}}^{\mathrm{fac}}=b_{2} \lambda_{\bar{q}} T^{4},
$$

where $a_{2} \equiv 8 \pi^{2} / 15$ and $b_{2} \equiv 7 \pi^{2} N_{f} / 40$. The equation of state (9) remains valid.

In Fig. 11 we show the ratio $\epsilon_{i}^{\mathrm{fac}} / \epsilon_{i}$ for quarks and gluons as a function of the fugacity $\lambda_{i}$. This ratio does not depend on the temperature. For bosons, the factorized expression tends to overestimate the correct result, for fermions, it underestimates it. In the interval $0 \leq \lambda_{i} \leq 1$, the error is maximized at $\lambda_{i}=0$, and decreases monotonically as $\lambda_{i} \rightarrow 1$. At $\lambda_{i}=0$, it is $8 \%$ for bosons, and $5 \%$ for fermions.

Energy and momentum is locally conserved,

$$
\partial_{\mu} T^{\mu \nu}(x)=0
$$

With (3), these are the equations of ideal fluid dynamics, which can be used to compute the evolution of the energy density and the fluid 4-velocity of the system. The fluid-dynamical equations (11) are closed by specifying the equation of state of matter under consideration, i.e., the pressure as a function of energy density, $p=p(\epsilon)$. In our case, this equation of state is simple, see eq. (9). 


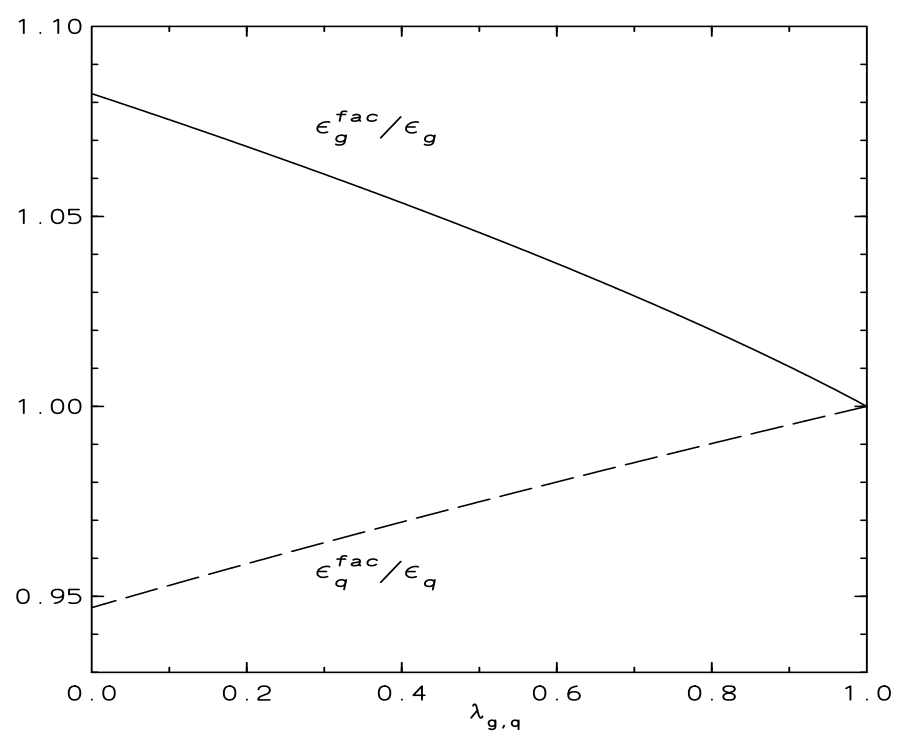

Figure 1: The ratio $\epsilon_{i}^{\mathrm{fac}} / \epsilon_{i}$ for gluons (solid line) and quarks (dashed line) as a function of the fugacity $\lambda_{i}$.

\subsection{Rate equations}

The 4-current of the number of particles of species $i$ is

$$
N_{i}^{\mu}(x) \equiv \int \frac{\mathrm{d}^{3} \mathbf{k}}{E_{i}} k^{\mu} f_{i}(x, k \cdot u)=n_{i}(x) u^{\mu}(x),
$$

where

$$
n_{i}(x) \equiv \int \mathrm{d}^{3} \mathbf{k} f_{i}\left(x, E_{i}\right)
$$

is the number density of particle species $i$ in the rest frame of a fluid element. This density is controlled by the value of the fugacity, $\lambda_{i}$. In general, the fugacities for the different particle species are independent thermodynamic variables, and one has to specify additional equations of motion which determine the number densities. These are the rate equations. To close the coupled set of fluid-dynamical equations and rate equations, one has to be specify an equation of state which, in general, also depends on the number densities of the various particle species, $p=p\left(\epsilon, n_{1}, n_{2}, \ldots\right)$.

The reason why there is actually no such dependence for the system considered here, eq. (9), is that the quarks and gluons are considered to be massless. This has the important consequence that, in our case, chemical non-equilibrium does not affect the evolution of the energy and momentum densities of the fluid! This certainly changes in the case of a nonzero strange quark mass, although, for large temperatures $T \gg m_{s}$, the dependence of the pressure $p$ on $n_{s}$ is relatively weak.

The number densities of gluons, quarks, and antiquarks in the rest frame of a fluid element are

$$
\begin{aligned}
& n_{g}=\lambda_{g} T^{3} \frac{d_{g}}{2 \pi^{2}} \int_{0}^{\infty} \mathrm{d} z \frac{z^{2}}{e^{z}-\lambda_{g}} \\
& n_{q}=\lambda_{q} T^{3} \frac{d_{q}}{2 \pi^{2}} \int_{0}^{\infty} \mathrm{d} z \frac{z^{2}}{e^{z}+\lambda_{q}}
\end{aligned}
$$




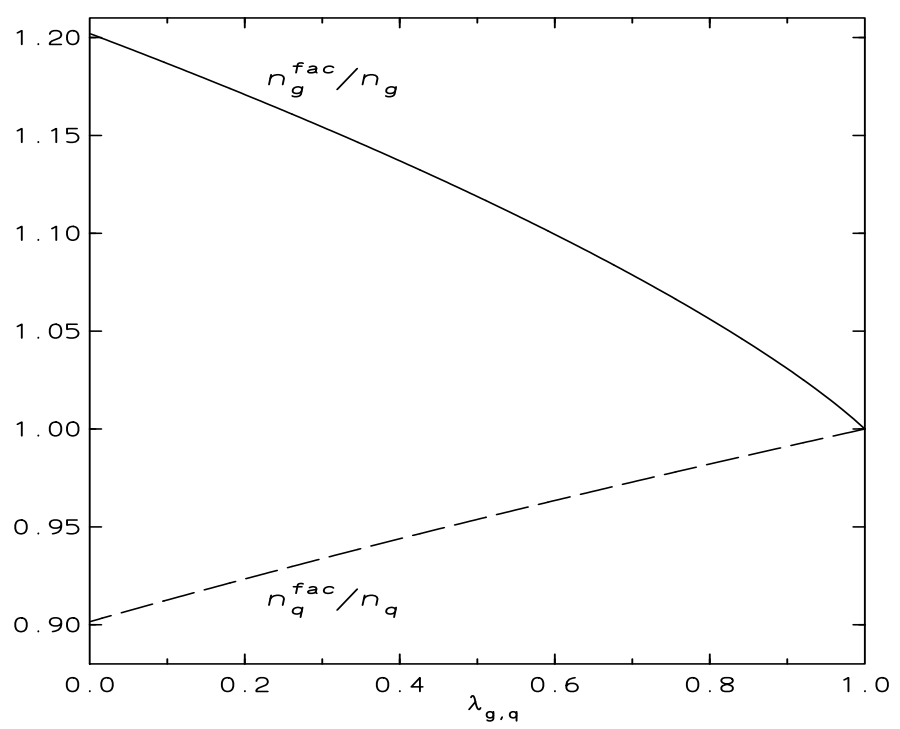

Figure 2: The ratio $n_{i}^{\mathrm{fac}} / n_{i}$ for gluons (solid line) and quarks (dashed line) as a function of the fugacity $\lambda_{i}$.

$$
n_{\bar{q}}=\lambda_{\bar{q}} T^{3} \frac{d_{q}}{2 \pi^{2}} \int_{0}^{\infty} \mathrm{d} z \frac{z^{2}}{e^{z}+\lambda_{\bar{q}}} .
$$

With the factorized distribution function (2) these expressions simplify to

$$
n_{g}^{\mathrm{fac}}=a_{1} \lambda_{g} T^{3}, \quad n_{q}^{\mathrm{fac}}=b_{1} \lambda_{q} T^{3}, \quad n_{\bar{q}}^{\mathrm{fac}}=b_{1} \lambda_{\bar{q}} T^{3},
$$

where $a_{1} \equiv 16 \zeta(3) / \pi^{2}$ and $b_{1} \equiv 9 \zeta(3) N_{f} /\left(2 \pi^{2}\right)$.

In Fig. 2, we show the ratio $n_{i}^{\text {fac }} / n_{i}$ as a function of fugacity. As for the ratio of energy densities, Fig. 1, this ratio does not depend on the temperature, and the factorized expression overestimates the correct result for bosons, while it underestimates it for fermions. Again, the error decreases as a function of $\lambda_{i}$, but it is about twice as large as for the energy densities. The maximum error (at $\lambda_{i}=0$ ) is about $20 \%$ for gluons and $10 \%$ for quarks.

In the absence of chemical equilibrium, the number of particles of species $i$ is determined from the rate equation

$$
\partial_{\mu} N_{i}^{\mu}(x)=R_{i}(x) .
$$

In chemical equilibrium, $R_{i}$ vanishes. In our case of a quark-gluon system, we assume that the $R_{i}$ are determined by the reactions $g g \leftrightarrow g g g$ and $g g \leftrightarrow q \bar{q}$. The rate equations (18) can then be written in the form [6]

$$
\begin{aligned}
& \partial_{\mu}\left(n_{g} u^{\mu}\right)=\mathrm{R}_{3} n_{g}\left(1-\frac{n_{g}}{\tilde{n}_{g}}\right)-2 \mathrm{R}_{2} n_{g}\left(1-\frac{n_{q} n_{\bar{q}} \tilde{n}_{g}^{2}}{\tilde{n}_{q} \tilde{n}_{\bar{q}} n_{g}^{2}}\right), \\
& \partial_{\mu}\left(n_{q} u^{\mu}\right)=\mathrm{R}_{2} n_{g}\left(1-\frac{n_{q} n_{\bar{q}} \tilde{n}_{g}^{2}}{\tilde{n}_{q} \tilde{n}_{\bar{q}} n_{g}^{2}}\right), \\
& \partial_{\mu}\left(n_{\bar{q}} u^{\mu}\right)=\mathrm{R}_{2} n_{g}\left(1-\frac{n_{q} n_{\bar{q}} \tilde{n}_{g}^{2}}{\tilde{n}_{q} \tilde{n}_{\bar{q}} n_{g}^{2}}\right),
\end{aligned}
$$


where the $\tilde{n}_{i}$ are the number densities computed at the same temperature $T$, but for $\lambda_{i}=1$. The terms $R_{2}$ and $R_{3}$ are given by [6]

$$
\mathrm{R}_{2} \simeq 0.24 N_{f} \alpha_{s}^{2} \lambda_{g} T \ln \left(1.65 / \alpha_{s} \lambda_{g}\right), \quad \mathrm{R}_{3} \simeq 2.1 \alpha_{s}^{2} T \sqrt{2 \lambda_{g}-\lambda_{g}^{2}},
$$

with $\alpha_{s}$ being the strong coupling constant.

We take $\alpha_{s}=0.3$ throughout this analysis, unless otherwise stated. Note that, while the densities on the left-hand side of eqs. (19) - (21) in general contain the full phasespace distribution function (1), the right-hand sides of these equations and the terms (22) are derived assuming the factorized distribution (2) [6]. This is in principle inconsistent. At least close to equilibrium, however, the right-hand sides of the rate equations (19) (21) are small, and we are allowed to neglect this inconsistency. On the other hand, far away from equilibrium we expect other contributions to the chemical reaction rates to be more important than the difference between factorized and full distribution functions. Such contributions are, for instance, multi-gluon processes $g g \rightarrow n g$ [13]. In this work, we only include the lowest-order rates (22).

In chemical equilibrium, the right-hand sides of the rate equations (19) - (21) vanish by definition, and we obtain

$$
\partial_{\mu}\left(n_{i}^{\mathrm{eq}} u^{\mu}\right)=0, \quad i=g, q, \bar{q} .
$$

We can now derive a relation for the time evolution of the ratio of the parton number density $n_{i}$ to its corresponding equilibrium value $n_{i}^{\text {eq }}$. By writing $n_{i}=n_{i}^{\text {eq }} n_{i} / n_{i}^{\text {eq }}$ on the left-hand sides of the rate equations (19) - (21), we derive with eq. (23) the condition

$$
u^{\mu} \partial_{\mu}\left(\frac{n_{i}}{n_{i}^{\mathrm{eq}}}\right)=\frac{R_{i}}{n_{i}^{\mathrm{eq}}} .
$$

This means that, for $R_{i} / n_{i}^{\mathrm{eq}}>0$, the comoving time derivative of the ratio of density to equilibrium density is positive, and thus this ratio is bound to grow with increasing proper time in each fluid element. Vice versa, this ratio decreases with time if $R_{i} / n_{i}^{\text {eq }}<0$. In other words, in the rest frame of a fluid element, $n_{i} / n_{i}^{\text {eq }}$ cannot decrease (increase) if $R_{i} / n_{i}^{\mathrm{eq}}>0(<0)$.

\subsection{Entropy}

Local thermodynamical equilibrium implies the conservation of entropy. If the system is not chemically equilibrated, the entropy is bound to grow, until chemical equilibrium is reached [7, 14]. To see this, we make the following observation: the form of the distribution function (11) or (2) is exactly that of a distribution function in local thermodynamical equilibrium, except that the fugacities, $\lambda_{i}$, assume values which are not the same as in chemical equilibrium, $\lambda_{i}^{\text {eq }}$. Note that in this case there are no further dissipative terms [15] in the tensor decomposition of (3) and (12] [16]. Nevertheless, the system is not in thermodynamical equilibrium, and the actual values of $\epsilon, p$, and $n_{i}$ will differ from the equilibrium values, $\epsilon^{\mathrm{eq}}, p^{\mathrm{eq}}$, and $n_{i}^{\mathrm{eq}}$.

Since the distribution function has the same form as in thermodynamical equilibrium, we can imagine each fluid element to actually be in chemical equilibrium with a (local) particle 
bath which determines the value of the fugacities instead of the rate equations (18). In other words, we can imagine local changes of particle number to be induced by a change of the parameters of the particle bath instead by chemical reactions within the fluid element itself. As long as these changes happen faster than those of the macroscopic fluid variables, one can view the fluid element to remain in (local) thermodynamic equilibrium, and apply thermodynamical relationships.

The assumption that microscopic reaction rates are much larger than the rate of change of macroscopic fluid variables is certainly an over-idealization in view of the characteristic time scales in relativistic heavy-ion collisions. So is, in fact, the ideal fluid limit which assumes complete (local) thermodynamical equilibrium. Nevertheless, our point of view is that ideal fluid dynamics still offers valuable information about the collective behavior of the system. Our approach is the simplest possible extension of ideal fluid dynamics. A better approximation would be to solve the equations for dissipative relativistic fluid dynamics in the presence of chemical reactions [17]. In general, chemical non-equilibrium gives rise to additional diffusion terms which do not appear in our treatment. So far, the complexity of these equations has discouraged attempts to apply them to the description of nuclear collision dynamics.

Accepting these caveats, we now proceed by contracting (11) with $u_{\nu}$,

$$
0=u_{\nu} \partial_{\mu} T^{\mu \nu}=u \cdot \partial \epsilon+(\epsilon+p) \partial \cdot u
$$

and use the first law of thermodynamics,

$$
\mathrm{d} \epsilon=T \mathrm{~d} s+\sum_{i} \mu_{i} \mathrm{~d} n_{i}
$$

( $s$ is the entropy density), as well as the fundamental relation of thermodynamics,

$$
\epsilon+p=T s+\sum_{i} \mu_{i} n_{i}
$$

to derive

$$
0=T(u \cdot \partial s+s \partial \cdot u)+\sum_{i} \mu_{i}\left(u \cdot \partial n_{i}+n_{i} \partial \cdot u\right)
$$

Equations (18) and (12), together with the definition of $\lambda_{i}$, can be used to conclude that

$$
\partial_{\mu} S^{\mu}=-\sum_{i} \ln \lambda_{i} R_{i}
$$

where $S^{\mu}=s u^{\mu}$. In chemical equilibrium, $R_{i}$ vanishes, and entropy is conserved.

Consider now the case of gluons, or quarks when the net-baryon number is zero. In this case, the equilibrium value of the fugacity $\lambda_{i}^{\mathrm{eq}}=1$, i.e., $\mu_{i}^{\mathrm{eq}}=0$. Now assume that the actual particle number density $n_{i}$ is smaller than the equilibrium value, corresponding to $\lambda_{i}<\lambda_{i}^{\mathrm{eq}}=1$. The rate equations drive $n_{i}$ towards equilibrium, i.e., the right-hand side has to be positive, $R_{i}>0$, in order to produce particles of species $i$. Since $\ln \lambda_{i}<0$, the change in entropy is positive. On the other hand, if $n_{i}$ is larger than in equilibrium, $\lambda_{i}>\lambda_{i}^{\mathrm{eq}}=1$, the rate equations reduce the number of particles of species $i$, i.e., the right-hand side is negative, $R_{i}<0$. Again, entropy increases, since $\ln \lambda_{i}>0$. 
Now suppose that $\lambda_{i}^{\text {eq }}>1$, for instance for quarks when the net-baryon number is positive. Naively, one would think that the previous argument fails in this case, and one might worry whether this could lead to a situation where entropy actually decreases towards its equilibrium value, which contradicts the second law of thermodyamics. This is, however, not the case. In a relativistic system, one can create quarks only in pairs with antiquarks. In order to conserve the net-baryon number, $\partial_{\mu}\left(N_{q}^{\mu}-N_{\bar{q}}^{\mu}\right) \equiv 0$, the right-hand side of the rate equation for the quark number density, $R_{q}$, must be equal to the right-hand side of the rate equation for the antiquark number density, $R_{\bar{q}}$, see eqs. (33) - (34) below.

In eq. (29), these two terms in the sum over $i$ can then be combined to $-\left(\mu_{q}+\mu_{\bar{q}}\right) R_{q} / T$. In equilibrium, $\mu_{q}^{\mathrm{eq}}=-\mu_{\bar{q}}^{\mathrm{eq}}$, such that $\mu_{q}+\mu_{\bar{q}}=\delta \mu_{q}+\delta \mu_{\bar{q}}$, where $\delta \mu_{i} \equiv \mu_{i}-\mu_{i}^{\text {eq }}$. If the quark (and consequently, the antiquark) number density is smaller than its equilibrium value, $\delta \mu_{q}+\delta \mu_{\bar{q}}<0$, the right-hand side of the rate equation is positive, $R_{q}>0$. Consequently, the sum of the quark and antiquark term on the right-hand side of (29) is positive, leading again to an increase in entropy. The argument is similar in the case when the quark number density is larger than in equilibrium, or when $\lambda_{i}^{\text {eq }}<1$. In all cases, the entropy increases.

\section{Initial conditions}

In order to solve the macroscopic transport equations discussed in the last section, one has to specify the initial conditions. In this paper, we use results from two different approaches, both for RHIC and LHC energies.

The first approach is the SSPC model employed in [7, 8]. We decided to use these initial conditions for two reasons. First, the values for initial energy and parton densities obtained in this approach constitute an upper bound of what is expected to be created in ultrarelativistic nuclear collisions at RHIC energies. Second, it allows us to directly compare the results of [7, 8] with ours and point out possible discrepancies.

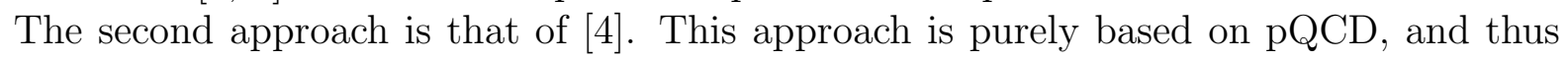
does not contain the contribution from the soft background, which constitutes about half of the total transverse energy at RHIC energies. In this sense, at RHIC this second approach constitutes a lower bound for the initial energy and parton number densities.

Table — contains the initial values for energy and parton number density for each approach and collision energy. The SSPC values are taken from [7], while the values shown for the pQCD approach [4] are computed from the $\mathrm{d} N / \mathrm{d} \eta$ and $\mathrm{d} E_{T} / \mathrm{d} \eta$ values near midrapidity $(-0.5 \leq \eta \leq 0.5)$ using a volume element $\Delta V=\pi R^{2} \tau_{0} \Delta \eta$ [18, such that

$$
\frac{\mathrm{d} E_{T}}{\mathrm{~d} \eta} \equiv \epsilon_{0} \frac{\mathrm{d} V}{\mathrm{~d} \eta}=\epsilon_{0} \tau_{0} \pi R^{2} \quad, \quad \frac{\mathrm{d} N_{i}}{\mathrm{~d} \eta} \equiv n_{i}^{0} \frac{\mathrm{d} V}{\mathrm{~d} \eta}=n_{i}^{0} \tau_{0} \pi R^{2} \quad, \quad i=g, q, \bar{q},
$$

where $R=1.12 A^{1 / 3} \mathrm{fm}$. From the four values $\epsilon_{0}, n_{g}^{0}, n_{q}^{0}$, and $n_{\bar{q}}^{0}$, using eqs. (5) and (14) - (16) one can unambiguously extract $T_{0}, \lambda_{g}^{0}, \lambda_{q}^{0}$, and $\lambda_{\bar{q}}^{0}$, and, from the latter, the values $n_{i}^{0} / \tilde{n}_{i}^{0}$ given in the last three columns of Table 1. 1 .

The initial time $\tau_{0}$ in the SSPC and pQCD approaches is chosen as $\tau_{0} \equiv 1 / p_{0}$, where $p_{0}$ is the infrared momentum cutoff required to regularize the mini-jet production cross sections. The values for $\tau_{0}$ given in Table 1 arise from the cutoffs $p_{0} \sim 0.8 \mathrm{GeV}$ for the SSPC approach and $p_{0} \sim 2 \mathrm{GeV}$ for the pQCD approach. The transverse momentum $p_{T} \geq p_{0}$ of a produced 
Table 1: Initial conditions for the fluid-dynamical expansion phase in central $A u+A u$ collisions at BNL RHIC and $P b+P b$ collisions at CERN LHC energies from the SSPC model and pQCD.

\begin{tabular}{|c|c|c|c|c|c|c|c|}
\hline Approach & Energy & $\begin{array}{c}\tau_{0} \\
(\mathrm{fm} / \mathrm{c})\end{array}$ & $\begin{array}{c}\epsilon_{0} \\
\left(\mathrm{GeV} / \mathrm{fm}^{3}\right)\end{array}$ & $\begin{array}{c}T_{0} \\
(\mathrm{GeV})\end{array}$ & $n_{g}^{0} / \tilde{n}_{g}^{0}$ & $n_{q}^{0} / \tilde{n}_{q}^{0}$ & $n_{\bar{q}}^{0} / \tilde{n}_{\bar{q}}^{0}$ \\
\hline SSPC & RHIC & 0.25 & 61.4 & 0.66 & 0.34 & 0.064 & 0.064 \\
SSPC & LHC & 0.25 & 425 & 1.01 & 0.43 & 0.082 & 0.082 \\
\hline pQCD & RHIC & 0.10 & 23.9 & 0.889 & 0.042 & 0.0077 & 0.0048 \\
pQCD & LHC & 0.10 & 1057 & 1.09 & 0.99 & 0.064 & 0.061 \\
\hline
\end{tabular}

mini-jet determines the time scale for the respective parton to come on mass-shell. Only after the partons are on their respective mass-shell, elastic scattering processes between them will drive the system towards kinetic equilibrium. How the system approaches kinetic equilibrium has to be studied in the framework of kinetic theory; our approach does not make any statements about this pre-equilibrium stage of a heavy-ion collision. In the following, we take $\tau_{0}=1 / p_{0}$, which is certainly the earliest possible time for the system to reach kinetic equilibrium. In the next section, we shall also use larger (and thus more conservative) values for $\tau_{0}$, in order to test the sensitivity of our results on this important parameter.

For RHIC initial conditions, the energy density in pQCD is much smaller than for the SSPC model, despite the smaller initial kinetic equilibrium time $\tau_{0}$. The reason is the absence of the soft background contribution in the former approach. For LHC, pQCD gives larger values for the initial energy density than the SSPC model. If scaled to the same initial proper time, however, the initial energy densities are approximately equal. This reflects the fact that the soft background contribution is rather small at LHC energies. Nevertheless, despite the similarity of the initial energy densities, in pQCD the gluons are close to chemical equilibrium at LHC energies, while this is not the case for the SSPC approach. Note that there is a small initial net-baryon number density $\left(n_{q}^{0}-n_{\bar{q}}^{0}\right) / 3$ in the pQCD approach, as opposed to the SSPC model where vanishing net-baryon number is assumed.

\section{Boost-invariant longitudinal expansion}

In this section, we study purely longitudinal expansion (in the $z$-direction) with boostinvariant initial conditions [18]. In this case, physics is constant along the space-time hyperbolae $\tau=\sqrt{t^{2}-z^{2}}=$ const.. Our aim is to establish how the parton equilibration process is affected when the full phase-space distribution (1) is used instead of the factorized distri- 
bution (8). We will furthermore compare the time evolution of the parton densities and the entropy in the SSPC and pQCD scenarios, and investigate the dependence of equilibration on the initial proper time $\tau_{0}$ and the strong coupling constant $\alpha_{s}$.

\subsection{Factorized vs. full phase-space distribution}

For boost-invariant longitudinal expansion, energy-momentum conservation (11) reads

$$
\dot{\epsilon}+\frac{\epsilon+p}{\tau}=0
$$

with $\dot{\epsilon} \equiv \mathrm{d} \epsilon / \mathrm{d} \tau, \tau$ being the proper time. The rate equations (19) - (21) assume the form

$$
\begin{aligned}
& \dot{n}_{g}+\frac{n_{g}}{\tau}=\mathrm{R}_{3} n_{g}\left(1-\frac{n_{g}}{\tilde{n}_{g}}\right)-2 \mathrm{R}_{2} n_{g}\left(1-\frac{n_{q} n_{\bar{q}} \tilde{n}_{g}^{2}}{\tilde{n}_{q} \tilde{n}_{\bar{q}} n_{g}^{2}}\right), \\
& \dot{n_{q}}+\frac{n_{q}}{\tau}=\mathrm{R}_{2} n_{g}\left(1-\frac{n_{q} n_{\bar{q}} \tilde{n}_{g}^{2}}{\tilde{n}_{q} \tilde{n}_{\bar{q}} n_{g}^{2}}\right) \\
& \dot{n}_{\bar{q}}+\frac{n_{\bar{q}}}{\tau}=\mathrm{R}_{2} n_{g}\left(1-\frac{n_{q} n_{\bar{q}} \tilde{n}_{g}^{2}}{\tilde{n}_{q} \tilde{n}_{\bar{q}} n_{g}^{2}}\right) \text {. }
\end{aligned}
$$

Equations (31) - (34) are four ordinary differential equations in the variable $\tau$, containing four unknowns, $T, \lambda_{g}, \lambda_{q}$, and $\lambda_{\bar{q}}$. They are numerically solved with a standard RungeKutta integration routine. At this point note that, while the evolution of the fluid energy and momentum densities are completely decoupled from the evolution of the parton densities on account of the equation of state (9), energy used up in parton production will be reflected by the temperature falling faster than in complete (local) thermodynamical equilibrium. In the latter case, $T \sim \tau^{-1 / 3}[18$.

For the factorized distribution, the evolution equations (31) - (34) can be further simplified [6]:

$$
\begin{aligned}
\frac{\dot{\lambda}_{g}+b\left(\dot{\lambda}_{q}+\dot{\lambda}_{\bar{q}}\right)}{\lambda_{g}+b\left(\lambda_{q}+\lambda_{\bar{q}}\right)}+4 \frac{\dot{T}}{T}+\frac{4}{3 \tau} & =0 \\
\frac{\dot{\lambda}_{g}}{\lambda_{g}}+\frac{3 \dot{T}}{T}+\frac{1}{\tau} & =\mathrm{R}_{3}\left(1-\lambda_{g}\right)-2 \mathrm{R}_{2}\left(1-\frac{\lambda_{q} \lambda_{\bar{q}}}{\lambda_{g}^{2}}\right) \\
\frac{\dot{\lambda}_{q}}{\lambda_{q}}+\frac{3 \dot{T}}{T}+\frac{1}{\tau} & =R_{2} \frac{a_{1}}{b_{1}}\left(\frac{\lambda_{g}}{\lambda_{q}}-\frac{\lambda_{\bar{q}}}{\lambda_{g}}\right) \\
\frac{\dot{\lambda}_{\bar{q}}}{\lambda_{\bar{q}}}+\frac{3 \dot{T}}{T}+\frac{1}{\tau} & =R_{2} \frac{a_{1}}{b_{1}}\left(\frac{\lambda_{g}}{\lambda_{\bar{q}}}-\frac{\lambda_{q}}{\lambda_{g}}\right)
\end{aligned}
$$

with $b=b_{2} / a_{2}=21 N_{f} / 64$.

We are now in a position to check how the equilibration process differs when using the factorized phase-space distribution function (2) as compared with the full distribution function (11). Fig. 3 shows the proper time evolution of quark and gluon number densities, $n_{q}$ and $n_{g}$, normalized to the corresponding values for $\lambda_{i}=1, \tilde{n}_{q}$ and $\tilde{n}_{g}$, for SSPC initial conditions at RHIC and LHC (cf. Table 1). The results for the factorized distribution function tend to slightly overestimate the degree of equilibration, but the deviation is of the order of a few percent only. This was to be expected from Fig. 2. 

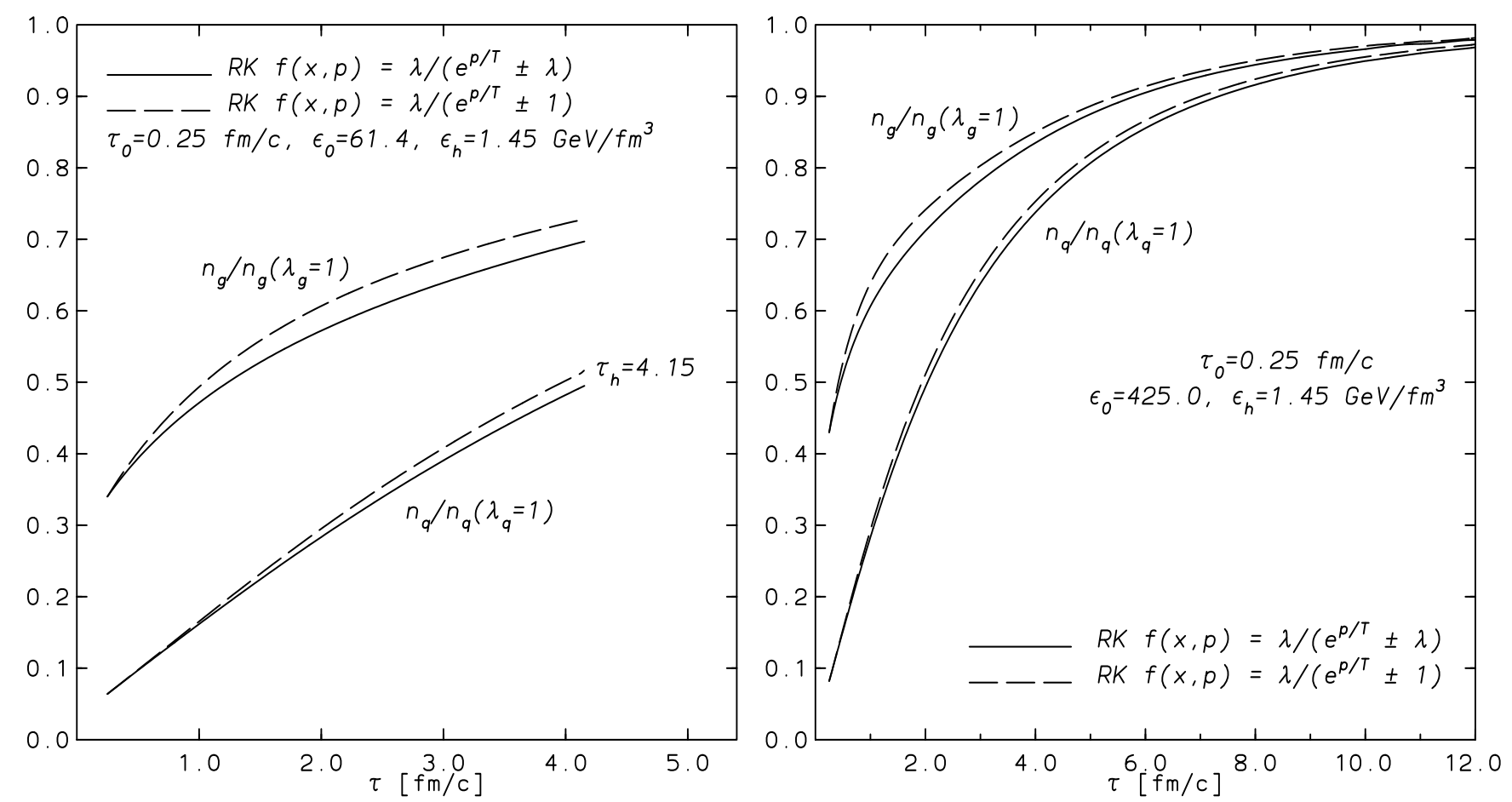

Figure 3: Parton densities for the SSPC RHIC (left) and LHC scenarios (right), normalized to the corresponding values for $\lambda_{i}=1$ (denoted as $\tilde{n}_{i}$ in the text). The solid line shows results for the full phase-space distribution function, while the dashed line those for the factorized distribution function $f^{\text {fac }}$. 

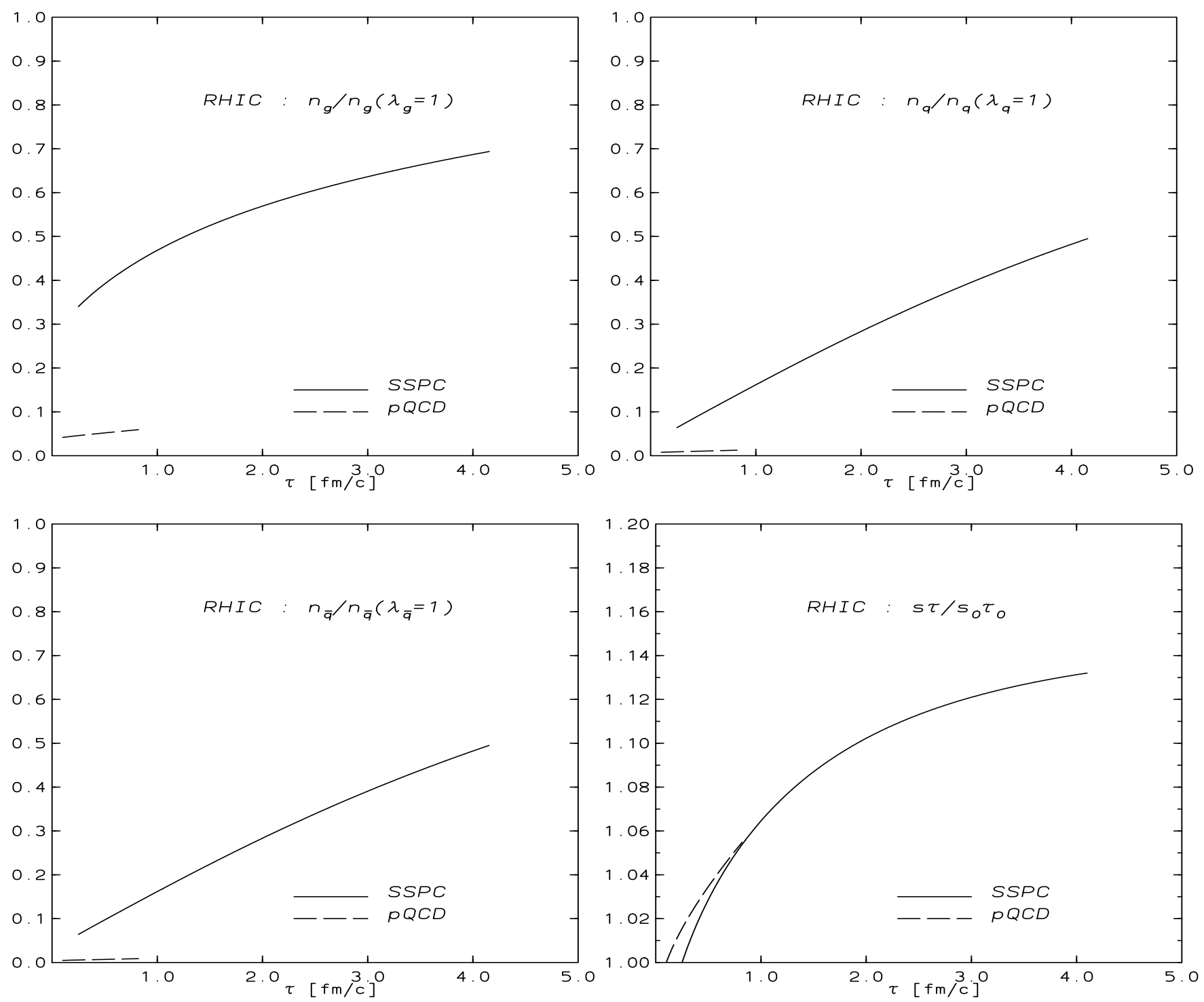

Figure 4: RHIC SSPC and pQCD scenarios (see Table 1): proper time evolution of the gluon (upper left panel), quark (upper right panel), and antiquark densities (lower left panel), and the entropy (lower right panel). The densities are normalized to their corresponding values for $\lambda_{i}=1$, the entropy $(\sim s \tau)$ to its initial value.

\subsection{Comparison of SSPC and pQCD scenarios}

We now compare the proper time evolution of the parton densities and the entropy in the SSPC and pQCD scenarios. We exclusively use the full distribution function (11) in the following. The entropy in a given rapidity unit is $\mathrm{d} S / \mathrm{d} \eta=s \tau \pi R^{2}$, where $\pi R^{2}$ is the transverse area of the expanding system. Since this area is constant in a purely longitudinal expansion, we may consider the product of entropy density and proper time, $s \tau$, as a measure for the entropy per rapidity unit.

In Fig. 1 we show the parton equilibration process for RHIC initial conditions (see Table 11), for the SSPC model (solid lines) and pQCD (dashed lines). For the SSPC case, the hadronization energy density $\epsilon_{h}=1.45 \mathrm{GeVfm}^{-3}$ is reached at a proper time $\tau_{h}=4.15 \mathrm{fm} / \mathrm{c}$, at which we stop the time evolution. The relative gluon density, $n_{g} / \tilde{n}_{g}$, reaches about 0.7 , and the relative quark density, $n_{q} / \tilde{n}_{q}$, about 0.5 . The entropy increases $13 \%$ before hadroniza- 

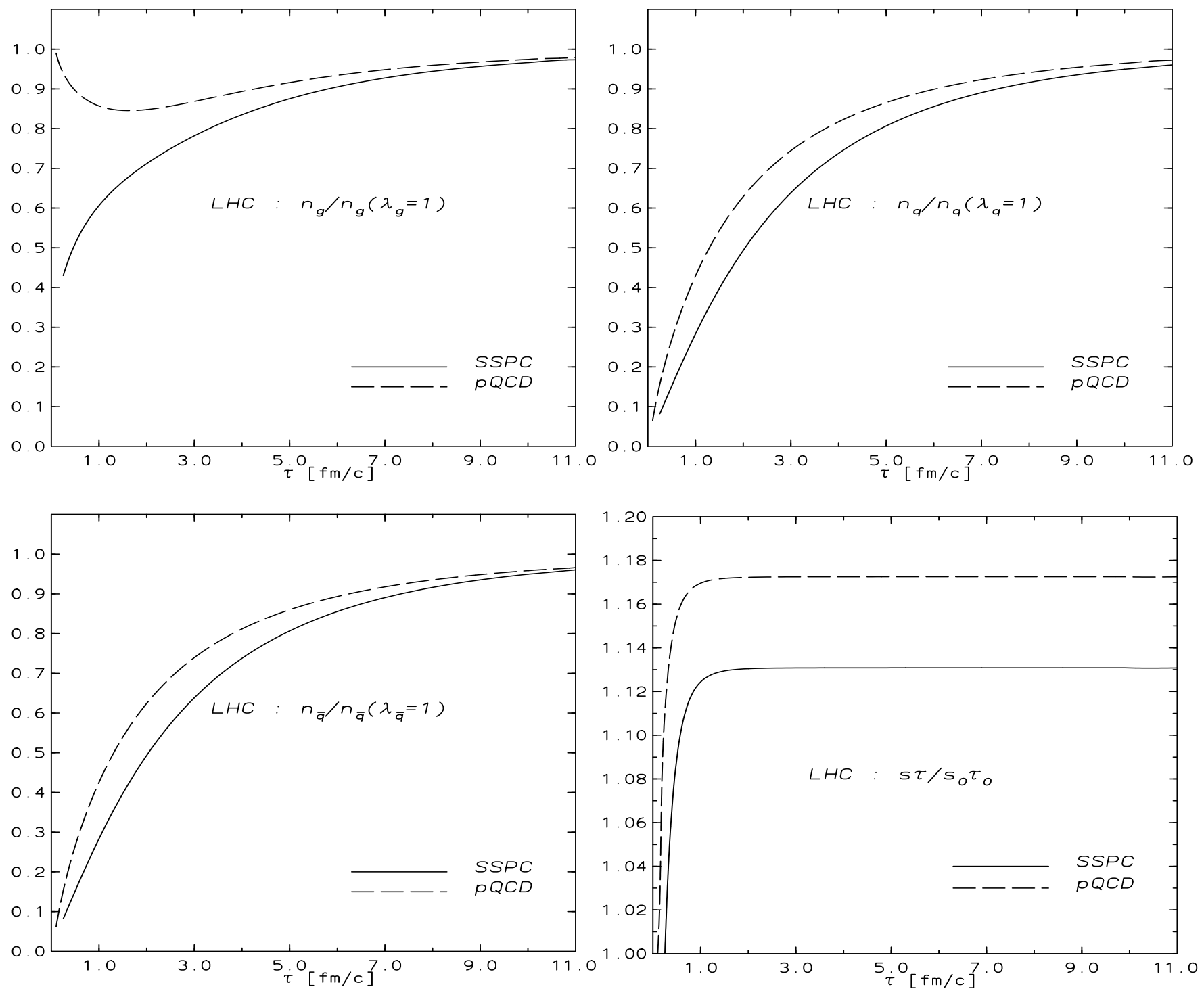

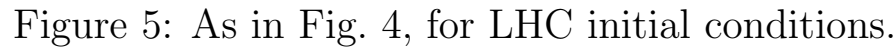

tion. For pQCD initial conditions, the QGP phase has little time to evolve, hadronizing only about $0.7 \mathrm{fm} / \mathrm{c}$ after thermalization. The partons have no time to equilibrate, and the entropy increases only $\sim 5 \%$.

While the SSPC scenario was considered to be net-baryon free, the pQCD scenario shows a slight difference in the quark and antiquark initial densities, corresponding to an initial baryon number density of about $0.12 \mathrm{fm}^{-3}$. We checked that our Runge-Kutta solver respects baryon number conservation, which in the purely longitudinally expanding geometry implies that the product $n_{B} \tau=\left(n_{q}-n_{\bar{q}}\right) \tau / 3$ is constant throughout the expansion. As a consequence of nonzero net-baryon number, the equilibrium ratio of $n_{q} / \tilde{n}_{q}$ will be larger than 1 , while that of $n_{\bar{q}} / \tilde{n}_{\bar{q}}$ will be smaller than 1 , because $\tilde{n}_{i}$ is computed with $\lambda_{i}=1$ instead of the correct equilibrium value $\lambda_{q}^{\mathrm{eq}}=1 / \lambda_{\bar{q}}^{\mathrm{eq}}>1$. For a small initial baryon number density of about $0.12 \mathrm{fm}^{-3}$, however, the deviation of $\lambda_{i}^{\text {eq }}$ from 1 is negligible.

The LHC case is shown in Fig. 5. The parton species are seen to approach equilibrium after about $10 \mathrm{fm} / \mathrm{c}$. We note that this time is well before a transverse rarefaction front can penetrate to the center of the QGP (see discussion in section 5). The reason why partons 
equilibrate is that the system starts at much higher temperature and higher initial values for the fugacities than at RHIC (see Table1). Particularly noteworthy is that, in the pQCD case, the gluons are almost completely equilibrated already at $\tau_{0}$, while the quarks and antiquarks are not. Consequently, quark-antiquark production processes drive the gluons temporarily out of equilibrium. Entropy production is of the order of $13 \%$ (SSPC) to $17 \%$ (pQCD).

\subsection{Sensitivity to $\tau_{0}$ and $\alpha_{s}$}

We now investigate the sensitivity of the equilibration process to variations of the initial proper time, $\tau_{0}$, and the strong coupling constant, $\alpha_{s}$. As discussed above, the values for the initial time $\tau_{0}$ in Table 1 are most certainly lower bounds. We therefore consider values $\tau_{0} \geq 0.25 \mathrm{fm} / \mathrm{c}$ for the $\mathrm{SSPC}$ scenario and $\tau_{0} \geq 0.1 \mathrm{fm} / \mathrm{c}$ for the pQCD approach. In varying $\tau_{0}$, we keep the produced transverse energy, $\mathrm{d} E_{T} / \mathrm{d} \eta$, and the parton numbers, $\mathrm{d} N_{i} / \mathrm{d} \eta$, constant. By eq. (30), the energy density and parton number densities must then decrease like $\sim 1 / \tau_{0}$. In essence, this means that we allow the system to evolve without doing longitudinal work. This is certainly an idealization: only when the pressure vanishes, the system does not perform any longitudinal work, but in our case pressure builds up as the system approaches kinetic equilibrium.

To facilitate the presentation, we only give the final values for the parton densities and the entropy at the end of the expansion of the QGP phase. The evolution of the QGP is terminated either at the hadronization time, $\tau_{h}$, when the longitudinal expansion has cooled the system down to an energy density $\epsilon_{h}=1.45 \mathrm{GeV} / \mathrm{fm}^{3}$, or at the rarefaction time, $\tau_{\text {rarefac }}$, when a transverse rarefaction wave reaches the center of the system (see discussion in section 5).

The time evolution equation for the energy density, (31), with the equation of state (9), has the solution $\epsilon / \epsilon_{0}=\left(\tau_{0} / \tau\right)^{4 / 3}$. According to eq. (30), the product $\epsilon_{0} \tau_{0}=\left(\mathrm{d} E_{T} / \mathrm{d} \eta\right) /\left(\pi R^{2}\right)$ is constant for constant transverse energy per unit rapidity and constant transverse area. Consequently, the hadronization time $\tau_{h}$ grows with the initial proper time $\tau_{0}$ according to $\tau_{h}=\tau_{0}^{1 / 4}\left[\left(\mathrm{~d} E_{T} / \mathrm{d} \eta\right) /\left(\pi R^{2} \epsilon_{h}\right)\right]^{3 / 4}$. The time spent in the QGP phase is therefore

$$
\Delta \tau \equiv \tau_{h}-\tau_{0}=\tau_{0}^{1 / 4}\left(\frac{\mathrm{d} E_{T} / \mathrm{d} \eta}{\pi R^{2} \epsilon_{h}}\right)^{3 / 4}-\tau_{0} .
$$

This time increases for small $\tau_{0}$, has a maximum at $\tau_{0}^{*}=\left(\mathrm{d} E_{T} / \mathrm{d} \eta\right) /\left(4^{4 / 3} \pi R^{2} \epsilon_{h}\right)$, and then decreases again. For the SSPC model at RHIC, $\tau_{0}^{*} \simeq 1.67 \mathrm{fm} / \mathrm{c}$, while for pQCD at RHIC, $\tau_{0}^{*} \simeq 0.27 \mathrm{fm} / \mathrm{c}$. At LHC energies, the values for $\tau_{0}^{*}$ are quite similar, for the SSPC model, $\tau_{0}^{*} \simeq 11.54 \mathrm{fm} / \mathrm{c}$, and for $\mathrm{pQCD}, \tau_{0}^{*} \simeq 11.48 \mathrm{fm} / \mathrm{c}$.

The hadronization time grows proportional to the initial transverse energy. For LHC energies, the transverse energy is so large that a transverse rarefaction wave (see section 5), travelling with sound velocity $c_{s}=1 / \sqrt{3}$ into matter at rest, reaches the center of the system before the longitudinal expansion has cooled matter down to $\epsilon_{h}$. At $z=0$, this transverse rarefaction wave reaches the center at time $\tau_{\text {rarefac }}=\tau_{0}+R / c_{s}$. For a $P b$ nucleus, $R=1.12 A^{1 / 3} \simeq 6.6 \mathrm{fm}$ and $\tau_{\text {rarefac }} \simeq \tau_{0}+11.5 \mathrm{fm} / \mathrm{c}$. While we use $\tau_{h}$ to terminate the time evolution at RHIC energy, for the LHC case, the time evolution is terminated at $\tau_{\text {rarefac }}$ instead of $\tau_{h}$. 

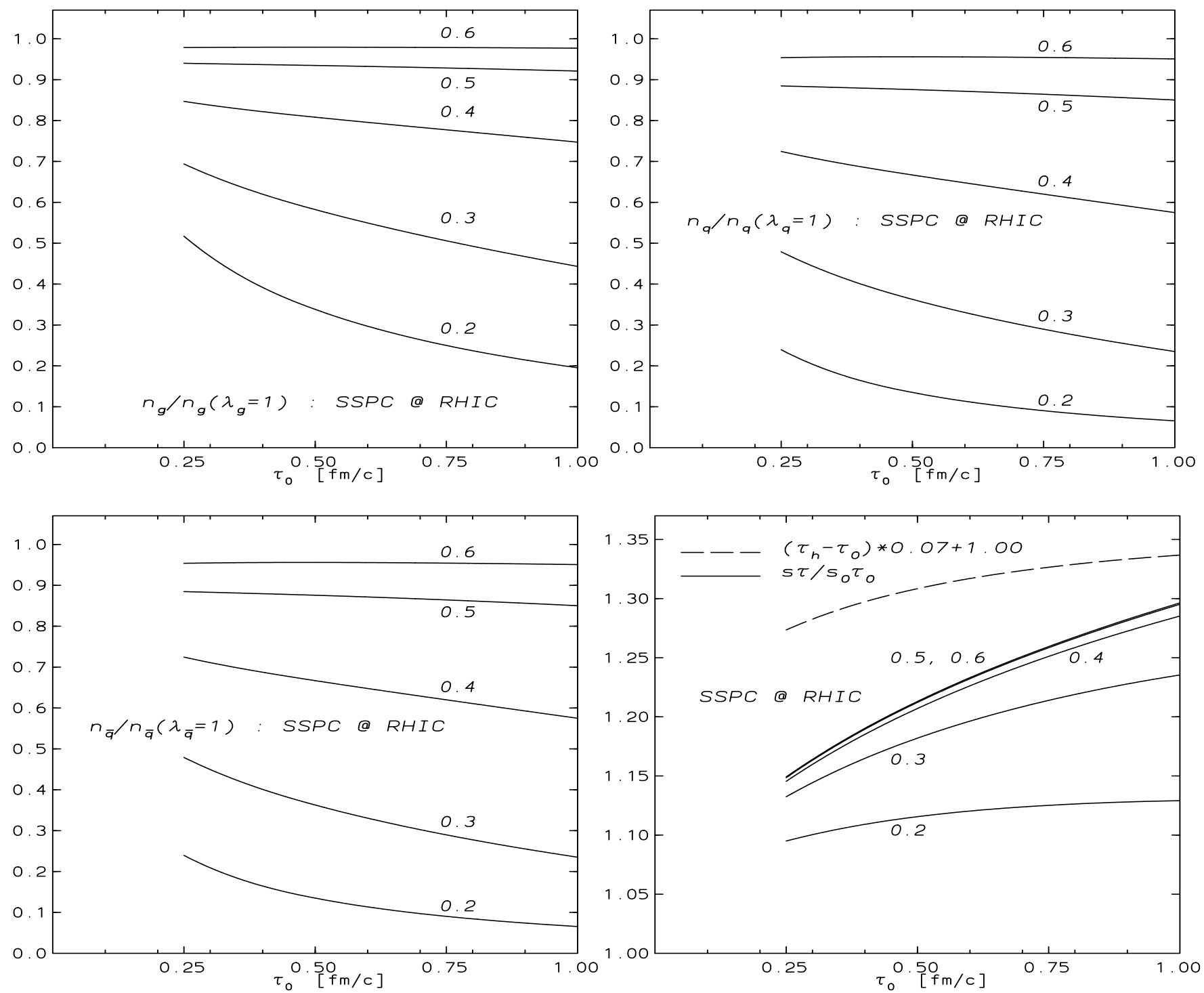

Figure 6: Hadronization values of the relative densities and the entropy for $\alpha_{s}=$ $0.2,0.3, \ldots, 0.6$, for the SSPC model with RHIC initial conditions. Upper left: gluons, upper right: quarks, lower left: antiquarks, lower right: $s \tau / s_{0} \tau_{0}$. The dashed line is the time the system spends in the QGP phase. 
Fig. 6 shows the case for the SSPC model with RHIC initial conditions. The hadronization values of the densities are closer to equilibrium for larger values of $\alpha_{s}$. This is obvious, since then the right-hand sides of the rate equations (32) - (34) are larger, cf. eq. (22), driving the system faster towards equilibrium. Consequently, also entropy production increases with $\alpha_{s}$.

On the other hand, the hadronization values of the densities are further away from equilibrium for increasing values of the initial time $\tau_{0}$. The reason is that, according to eq. (30), the values for the initial energy density and the parton densities decrease for increasing $\tau_{0}$ (the parton number rapidity density $\mathrm{d} N_{i} / \mathrm{d} \eta$ and transverse energy rapidity density $\mathrm{d} E_{T} / \mathrm{d} \eta$ are kept constant). Surprisingly, this does not have an effect on the initial temperature which is to all intents and purposes independent of $\tau_{0}$. To understand this, consider the factorized expressions (10) and (17); reducing energy and parton densities by the same factor is achieved by reducing the fugacities only, keeping the temperature constant. However, the reduction of the fugacities leads to smaller initial values for the parton densities. This puts the initial densities further away from their equilibrium values. This difference is, with increasing $\tau_{0}$, increasingly harder to overcome during the lifetime $\Delta \tau$, eq. (39), of the QGP phase. The amount of entropy produced in the RHIC scenarios is proportional to the lifetime of the QGP phase, i.e., the time during which chemical reactions drive the system towards thermodynamical equilibrium and thus increase the entropy. Note that equilibration is never complete at RHIC, unless one uses rather large values of $\alpha_{s}$.

The pQCD RHIC scenario is depicted in Fig. 7. The parton densities behave as in Fig.

6. The main quantitative difference is that, due to the small initial values of the energy and parton densities, even for large values of $\alpha_{s}$ the system never reaches chemical equilibrium. The behavior of the entropy follows again that of the lifetime of the QGP.

The LHC case is shown in Figs. 8 and 9. Despite different initial conditions, the relative densities at $\tau_{\text {rarefac }}$ are remarkably similar in both the SSPC model and pQCD. Again, this ratio increases with increasing $\alpha_{s}$ and decreasing $\tau_{0}$. The entropy increases with $\alpha_{s}$ and $\tau_{0}$. However, in this case the time the system spends in the QGP phase is constant, $\Delta \tau \equiv$ $\tau_{\text {rarefac }}-\tau_{0}=R / c_{s} \simeq 11.5 \mathrm{fm} / \mathrm{c}$ for $\mathrm{Pb}$. The increase in entropy can only be explained by the fact that the time integral over the right-hand side of eq. (29) is larger, if the system is further away from equilibrium (which is the case for larger values of $\tau_{0}$ ). As already seen in Fig. 5, entropy production is stronger in the pQCD case. Equilibration is nearly complete for large values of $\alpha_{s}$, independent of the value of $\tau_{0}$. The only scenario where the QGP will not reach full chemical equilibration is when $\alpha_{s}$ is small, and the initial time is large.

\section{$5 \quad$ Longitudinal and transverse expansion}

In this section, we consider (cylindrically symmetric) transverse expansion in addition to boost-invariant longitudinal expansion. In this case, the time evolution is no longer given by ordinary differential equations, and one has to resort to numerical algorithms which solve the equations of fluid dynamics, eqs. (11), and the rate equations (19) - (21). The numerical scheme to solve the fluid-dynamical equations used in this analysis is the relativistic HartenLax-Van Leer-Einfeldt (RHLLE) algorithm [19], with geometrical corrections performed using Sod's method [20]. Since the rate equations are conservation equations with source 

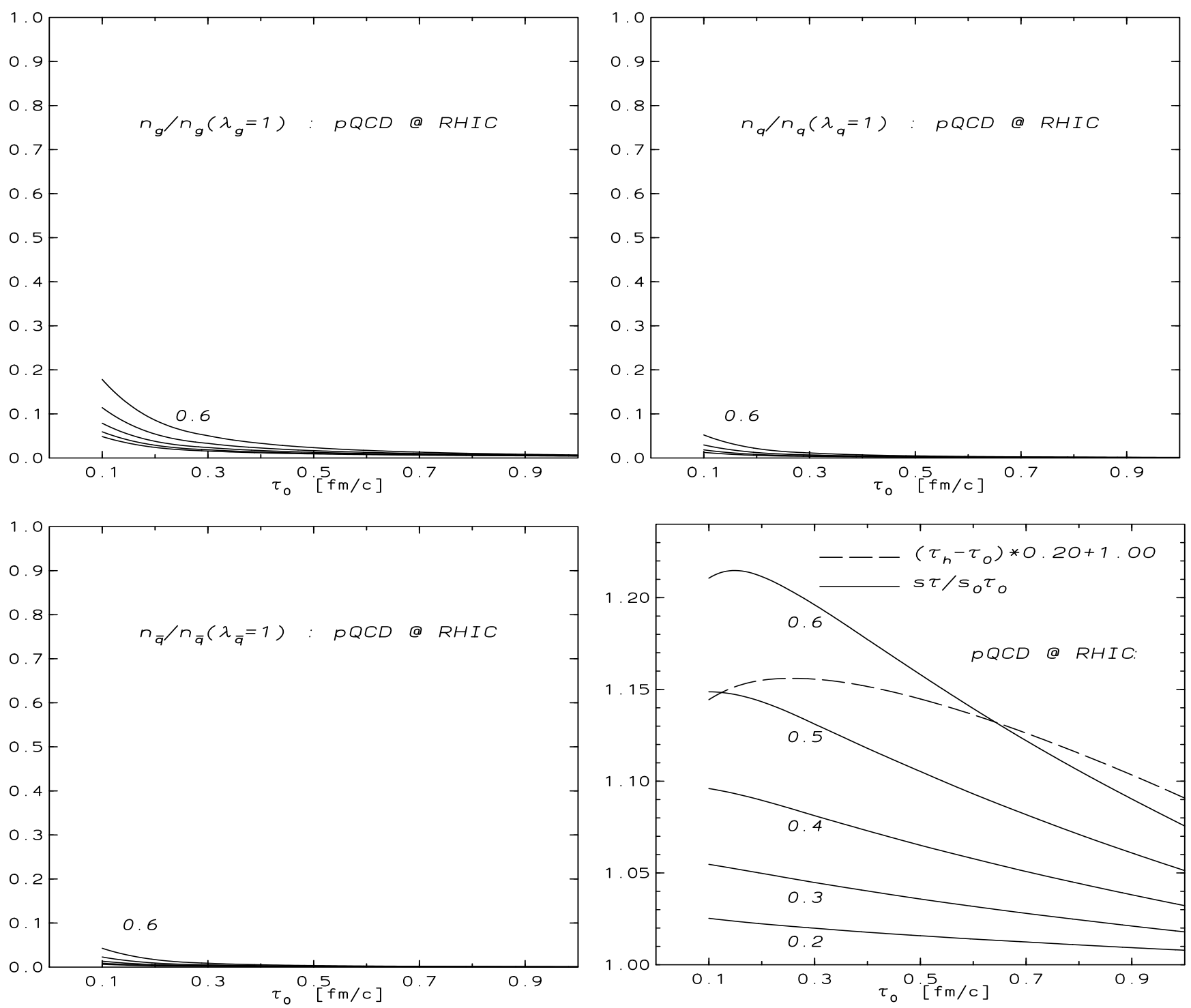

Figure 7: As in Fig. 6, for pQCD initial conditions. 

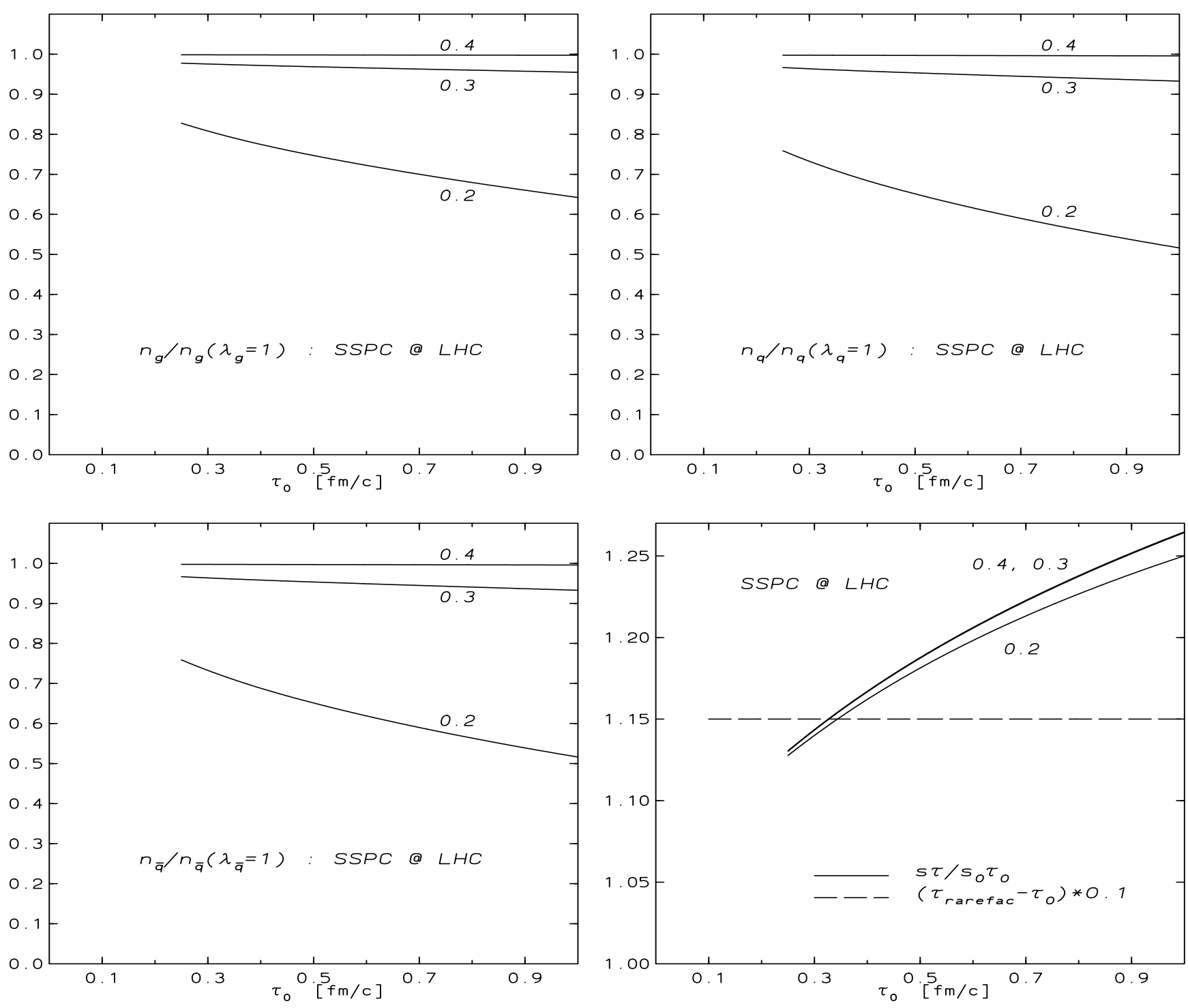

Figure 8: As in Fig. 6, for LHC initial conditions. The largest value of $\alpha_{s}$ taken here is 0.4; the curves for larger values are indistinguishable from this case. 

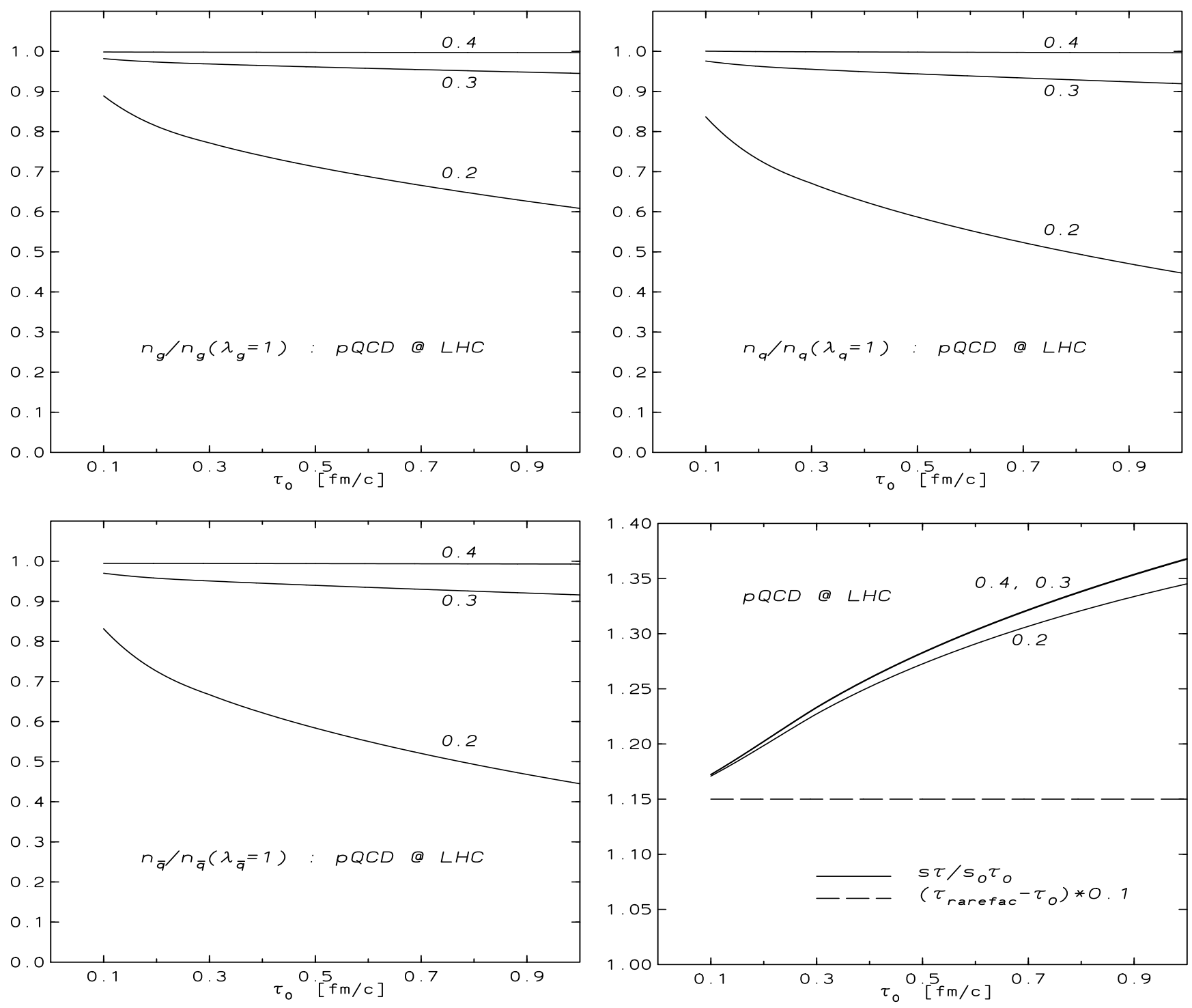

Figure 9: As in Fig. 8, for pQCD initial conditions. 
terms, they can also be solved with the RHLLE algorithm, with the right-hand sides being treated with Sod's method. Because of boost-invariance, it is sufficient to solve the equations in the central plane, $z=0$, where $\tau \equiv t$.

Our results are exclusively for the SSPC model and for the initial values given in Table 1; we did not vary $\tau_{0}=0.25 \mathrm{fm} / \mathrm{c}$ or $\alpha_{s}=0.3$. We first show results for a box profile $\sim \Theta(R-r)$,

and then for a wounded-nucleon profile $\sim 3 / 2 \sqrt{1-r^{2} / R^{2}}$. To facilitate comparison with [7], for the box profile we use $R=7 \mathrm{fm}$ both at RHIC and LHC energies. For the woundednucleon profile we use $R=1.12 A^{1 / 3}$ to compute the actual values for the radii of $A u$ nuclei (at RHIC) and $P b$ nuclei (at LHC).

\subsection{Box profile}

In Fig. 10 we show the evolution of the system in the $t-r$ plane for RHIC initial conditions. In order to highlight the consequences of transverse expansion and facilitate comparison with [7, 8], we plot constant energy contours $\epsilon(r, t)=\epsilon_{0} / N^{4 / 3}$. If there were no transverse expansion of the system, the energy contours for $N \geq 2$ would be parallel to the $N=1$ contour. We see that a rarefaction wave travels into the system from the surface, but does not reach the center before the interior cools below the hadronization energy density $\epsilon_{h}$ at $\tau_{h}=$ $4.15 \mathrm{fm} / \mathrm{c}$. This agrees with the Runge-Kutta analysis of the purely longitudinal expansion presented above. It disagrees with Fig. 1 of [7], where the hadronization time is of the order of $5 \mathrm{fm} / \mathrm{c}$. Upon hadronization, the relative gluon density, $n_{g} / \tilde{n}_{g}$, has reached roughly 0.7 , while the relative quark (and antiquark) density is $n_{q} / \tilde{n}_{q} \simeq 0.5$. Modest transverse velocities develop near the surface of the system. We find them to be smaller than those in Fig. 1 of [7]. This difference is due to the different numerical scheme used in [7] (the SHASTA algorithm of [21]; we use the RHLLE algorithm [19]). In contrast to [7], Fig. 1, we do not see that these velocities drive the fluid locally away from chemical equilibrium; in our case, the respective fluid elements continue to equilibrate.

Let us give a simple argument as to why this must be the case. Consider a fluid element on a given energy density contour in the $t-r$ plane (upper left panel of Fig. 10). Then, as the system expands, $\partial \cdot u>0$, the energy density drops as a function of proper time, $u \cdot \partial \epsilon=-(\epsilon+p) \partial \cdot u<0$. Since any other contour lying outside the original contour (relative to the origin) corresponds to a smaller energy density, it also corresponds to later proper times. Therefore, in going from the first contour to the second, the proper time in the rest frame of a fluid element increases. Since the actual parton number densities are smaller than their equilibrium values, and consequently the right-hand sides of the rate equations positive, $R_{i}>0$, we conclude from eq. (24) that $n_{i} / n_{i}^{\text {eq }}$ must increase in going from the first contour to the second. In order to compare with $n_{i} / \tilde{n}_{i}$, note that $\tilde{n}_{i}$ is computed at the same temperature $T$ as $n_{i}$, with the fugacity $\lambda_{i}$ set to 1 . On the other hand, while $\lambda_{i}^{\text {eq }}$ is also equal to 1 for the SSPC case considered here (on account of vanishing net-baryon density), the temperature $T^{\mathrm{eq}}$ in $n_{i}^{\mathrm{eq}}$ is in general different from $T$. Let us assume that the initial temperature for an evolution in complete local equilibrium is the same as that for the evolution including chemical equilibration. This has the consequence that initially $\tilde{n}_{i}=n_{i}^{\text {eq }}$, and thus $n_{i} / \tilde{n}_{i}=n_{i} / n_{i}^{\text {eq }}$ at $\tau_{0}$. As noted earlier in section 4.1 , in complete local equilibrium the temperature falls less rapidly than in chemical non-equilibrium, $T^{\mathrm{eq}}>T$ at all times $\tau>\tau_{0}$. Therefore, $n_{i} / \tilde{n}_{i}=\left(n_{i} / n_{i}^{\text {eq }}\right)\left(T^{\mathrm{eq}} / T\right)^{3} \geq n_{i} / n_{i}^{\mathrm{eq}}$, with the equality holding at 

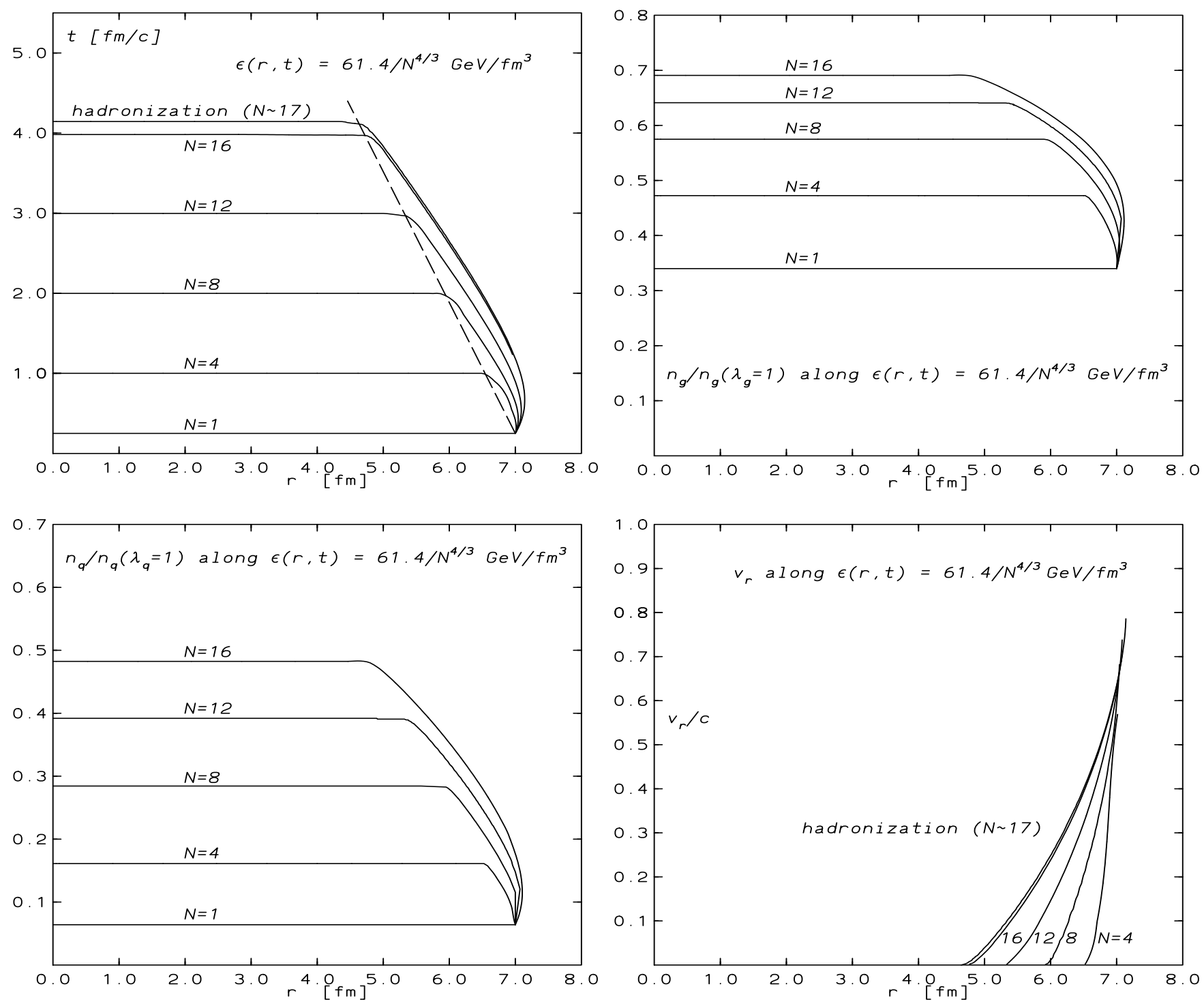

Figure 10: SSPC RHIC scenario: evolution in the $t-r$ plane. Upper left panel: energy density contours. The dashed line represents the advance of the rarefaction front. Upper right panel: relative density of gluons. Lower left panel: relative density of quarks (antiquarks identical). Lower right panel: transverse velocity.

$\tau_{0}$. Thus, not only does $n_{i} / \tilde{n}_{i}$ not decrease, it increases even faster than $n_{i} / n_{i}^{\text {eq }}$ during the evolution. Consequently, in the $n_{i} / \tilde{n}_{i}-r$ diagram (upper right and lower left panels of Fig. 10), the second contour must lie outside the first contour (with respect to the origin). This is exactly what we find numerically.

Fig. 11 shows the SSPC LHC scenario. In this case, a rarefaction wave has enough time to penetrate into the center of the system, albeit only shortly before the energy density falls to $\epsilon_{h}=1.45 \mathrm{GeV} / \mathrm{fm}^{3}$. Until the rarefaction wave reaches the center at proper time $\tau=\tau_{0}+R / c_{s}$, the evolution of the system at $r=0$ is given by a purely longitudinal expansion, as discussed in the previous section. Once again, the results obtained with the RHLLE algorithm agree well with the analysis using the Runge-Kutta method. As seen above, equilibration of the parton species is nearly complete.

The hadronization time agrees well with that found in [7], Fig. 3. However, in contrast to that analysis we again find that the transverse velocities developing in the rarefaction 

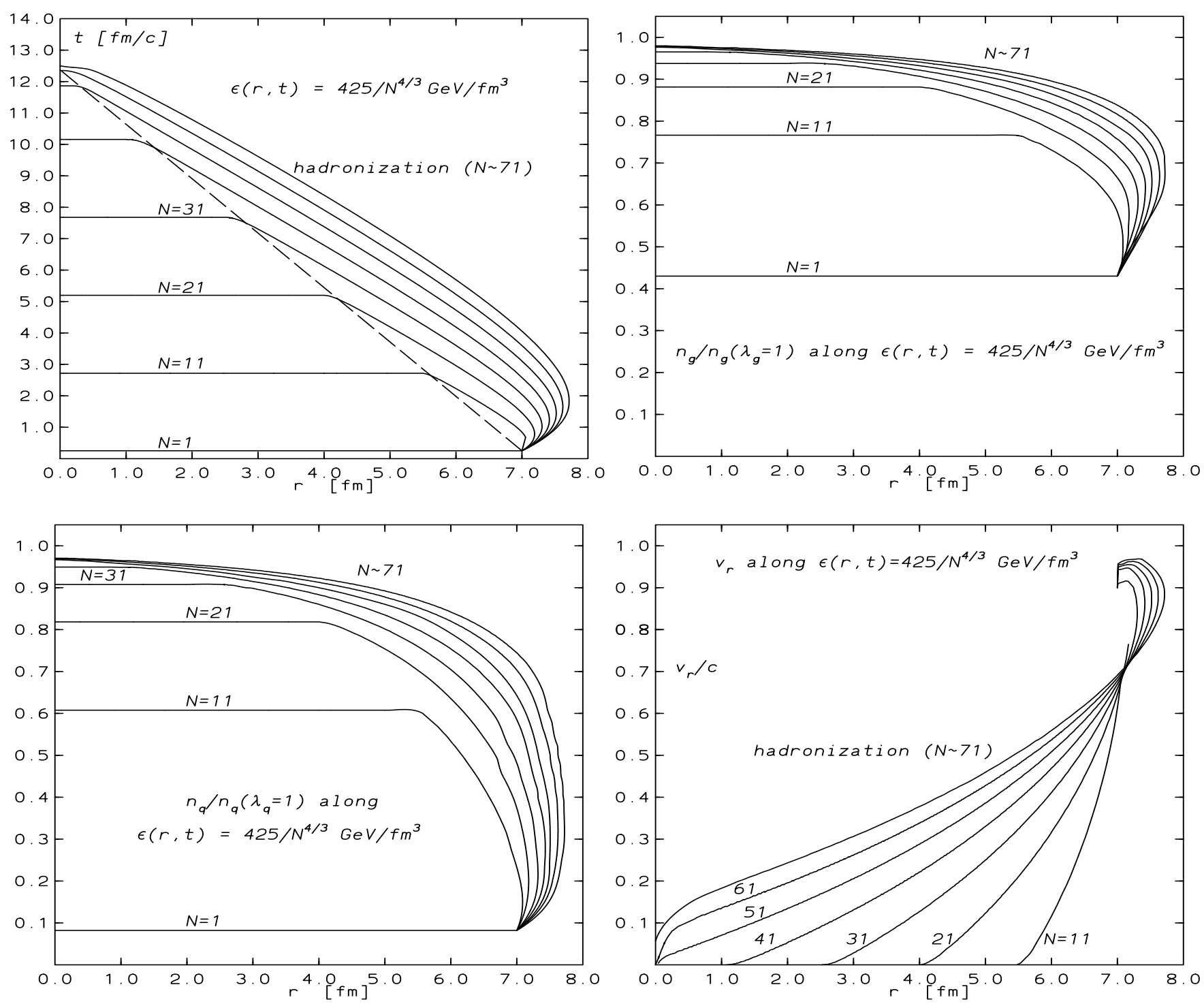

Figure 11: As in Fig. 10, for the SSPC LHC scenario. 
front do not impede chemical equilibration. In particular, we do not find the tendency to drive the system out of equilibrium at larger transverse distances $r$, and to overshoot the equilibrium values close to the origin, as in Fig. 3 of [7]. As explained above, in the $n_{i} / \tilde{n}_{i}-r$ diagram, contours corresponding to smaller energy density (larger proper times) must lie outside those corresponding to larger energy density (smaller proper times) relative to the origin.

\subsection{Wounded-nucleon profile}

Throughout the analysis presented above a box-profile function has been assumed. While this is instructive and facilitates direct comparison between the results from a purely longitudinal expansion and those including transverse expansion (as long as the transverse rarefaction wave has not yet reached the center of the system), a box profile is clearly an idealization.

In this section we repeat the analysis of the SSPC RHIC and LHC scenarios with a more realistic nuclear profile function, the so-called wounded-nucleon profile

$$
T_{A}(r)=\frac{3}{2} \sqrt{1-\frac{r^{2}}{R^{2}}},
$$

where $r$ is the transverse coordinate. We generate an $r$-dependence of our initial energy and parton density profiles by multiplying the initial values of Table 1 1 with eq. (40). Clearly, this implies higher initial densities at the center of the plasma. However, the fluid-dynamical expansion begins with a non-zero density gradient already at $\tau_{0}$, which, without the factor $3 / 2$ in (40), would lead to faster cooling than a constant nuclear profile function.

In Fig. 12 we present the analogue of Fig. 10, now computed with an initial woundednucleon density profile. We observe that the lifetime of the plasma phase is actually larger than in Fig. 10, $\tau_{h}=4.70 \mathrm{fm} / \mathrm{c}$, instead of $\tau_{h}=4.15 \mathrm{fm} / \mathrm{c}$. Due to the initial density gradients, transverse velocities develop now over the entire volume of the system. The cooling effects of the flow become apparent when considering purely longitudinal expansion with an initial energy density of $3 / 2 \times 61.4 \mathrm{GeV} / \mathrm{fm}^{3}$. In this case, the QGP lives longer, $\tau_{h}=5.63 \mathrm{fm} / \mathrm{c}$.

The increase of the initial energy and parton densities by a factor of $3 / 2$ in the center of the system has the effect that the partons at small and intermediate $r$ equilibrate a little further in the wounded-nucleon scenario than in the box-profile scenario. The relative gluon density reaches values around 0.8 ( 0.7 for the box profile) and the relative quark density values around 0.6 (compared with 0.5 in the box profile case). In general, however, the box-profile and the wounded-nucleon-profile relative parton density calculations agree to a remarkable extent.

In Fig. 13 we show the LHC case, where the effects of the wounded-nucleon profile become more apparent. The plasma cools markedly more rapidly than in the box-profile case, hadronizing at a proper time $\tau_{h}=9.37 \mathrm{fm} / \mathrm{c}$. This is more than $3 \mathrm{fm} / \mathrm{c}$ earlier than for an initial box profile, in which case $\tau_{h}$ is essentially moments after $\tau_{\text {rarefac }}$, the arrival of the rarefaction wave at $r=0$.

While the plasma's lifetime is significantly reduced, parton chemistry, driven by high temperatures and high gluon densities, is sufficiently rapid to equilibrate the plasma prior to hadronization. Qualitatively, the transverse velocities along contours of constant energy 

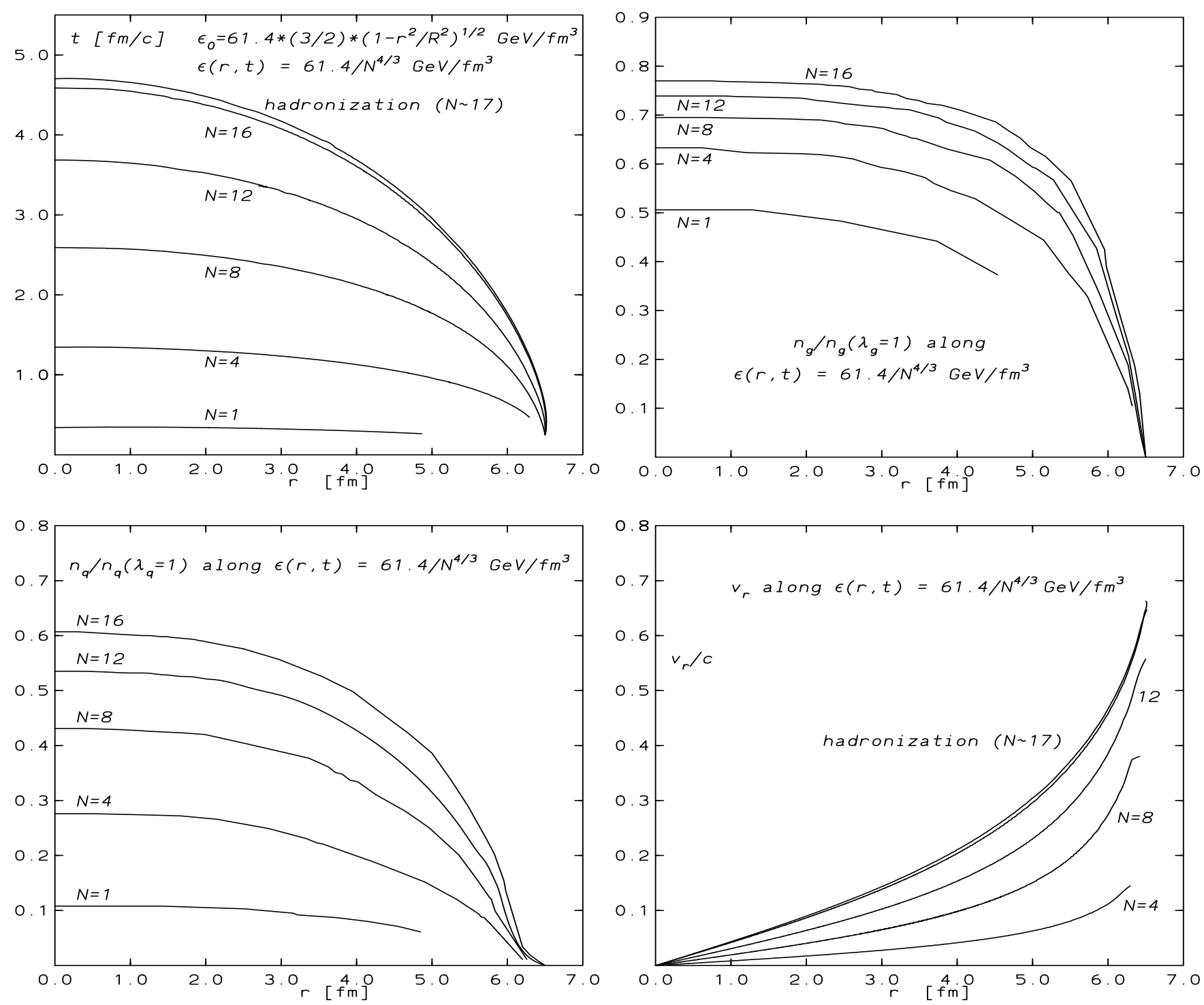

Figure 12: As in Fig. 10, with an initial wounded-nucleon profile. 

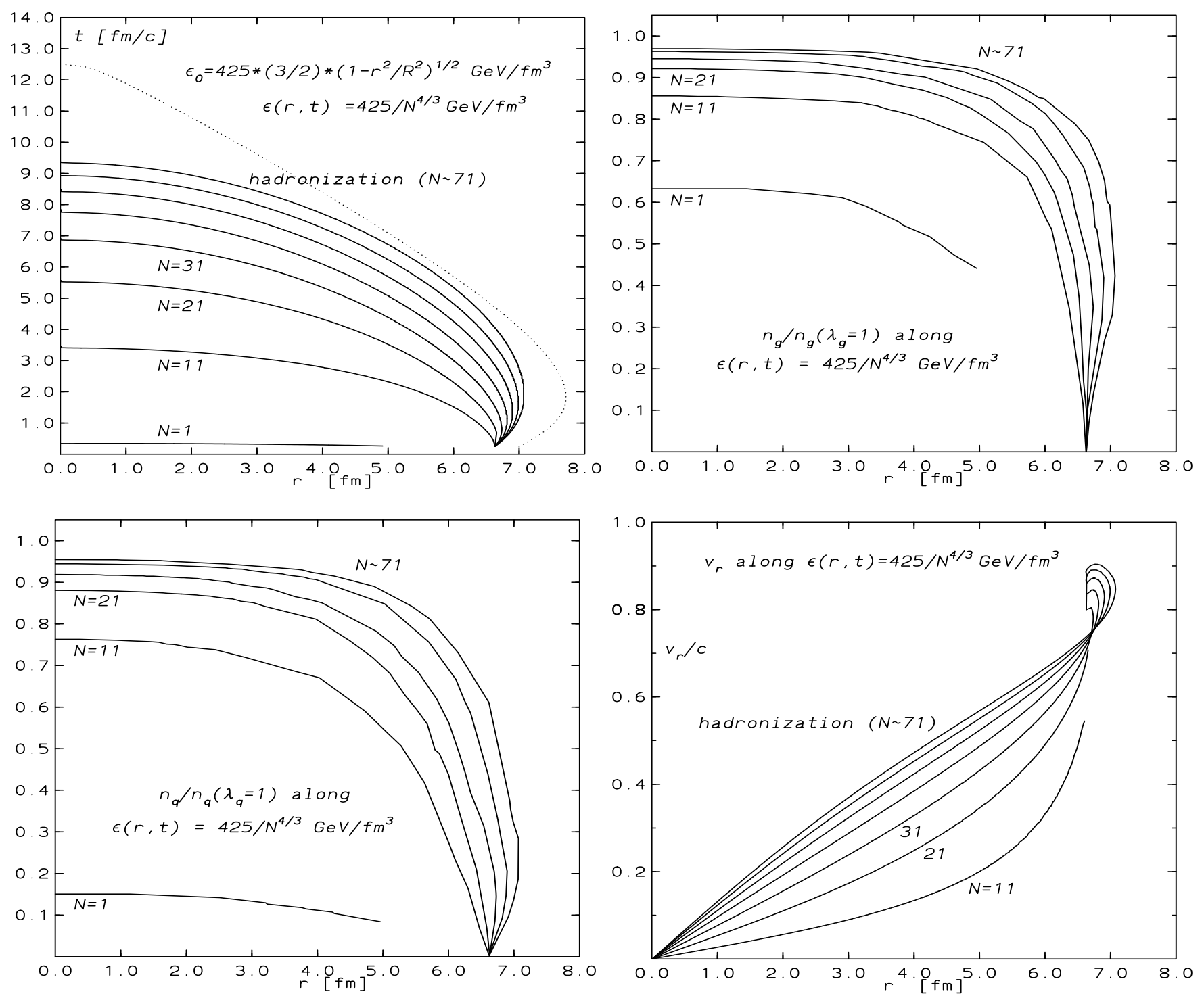

Figure 13: As in Fig. 11, with an initial wounded-nucleon profile. The dotted line in the upper left panel depicts the hadronization hypersurface for an initially box-profiled plasma (of $R=7 \mathrm{fm}$ ), shown in Fig. 11 . 
density in Figs. 12 and 13 agree with the analysis given in [8]. No parton evolution plots are shown in [8].

\section{Conclusions}

We have investigated chemical equilibration of quarks and gluons at RHIC and LHC energies. Assuming that a kinetically equilibrated quark-gluon plasma is created at these energies, we have used ideal fluid dynamics coupled to rate equations for the parton densities [6, 0, 8] to study chemical equilibration in the further evolution of the system. Our work is motivated by the upcoming experiments at RHIC in the fall of this year, and by recent pQCD estimates on parton production in the initial stage of ultrarelativistic nuclear collisions [4]. These estimates serve as initial conditions to the fluid-dynamical as well as the rate equations. Besides pQCD initial conditions, we also considered those given by the so-called SSPC model [7, 8], in order to compare our results to previous studies. At RHIC, the SSPC model constitutes an upper limit for the initial energy and parton densities, while pQCD sets a lower limit, as the effect of soft background fields is neglected in this approach. It was recently argued [22] that the transverse momentum cutoff $p_{0}$ should be chosen to match the saturation scale, $p_{\text {sat }} \sim 1 \mathrm{GeV}$ for RHIC, rather than $\sim 2 \mathrm{GeV}$ chosen here. Then, the initial conditions obtained from pQCD are rather similar to those in the SSPC approach. The produced transverse energy density can also be computed within classical Yang-Mills theory [23]; the values obtained in this way support the SSPC estimates.

Our results can be summarized as follows. Chemical equilibration of partons is never complete at RHIC energies. The highest degree of equilibration can be reached with SSPC initial conditions and an (unphysically) large value of the strong coupling constant, $\alpha_{s} \sim 0.6$. With initial conditions from pQCD, the system never comes close to equilibration.

The situation changes completely at LHC energies. Here, the initial energy and parton densities are already so large that chemical equilibration is complete in almost all scenarios considered. Only if $\alpha_{s} \sim 0.2$ or smaller and the initial proper time $\tau_{0}$ is large, equilibration was seen to be incomplete.

Within the macroscopic transport approach employed here, entropy production due to chemical non-equilibrium processes was found to be large, in some cases $\sim 30 \%$. This has implications for estimating the initial conditions in ultrarelativistic nuclear collisions. Commonly, one assumes that the evolution of the system is entropy conserving. Then, since $s \simeq$ const. $\times n$, the initial entropy $\sim s_{0} \tau_{0}$ is estimated from the total multiplicity of hadrons in the final state by the formula $\mathrm{d} N_{h} / \mathrm{d} \eta \simeq$ const. $\times s_{0} \tau_{0} \pi R^{2}$. However, if entropy grows due to chemical reactions, this formula would severely overestimate the entropy in the initial state.

We furthermore established that the use of a factorized phase-space distribution as in [6, 7, 8] is permissible as the error is of the order of a few percent only. We also gave an analytical proof, as well as numerical evidence, that transverse flow does not drive the system away from chemical equilibrium, in contrast to the results of $[7]$.

Future studies should focus on the further evolution of the system into the mixed and hadron phases. To this end, previously developed non-equilibrium models could be resurrected [24]. Further applications of the methods presented here include the study of 
strangeness and charm equilibration at RHIC and LHC energies [12]. Besides this, the reaction rates should be improved using the full phase-space distribution function instead of the factorized one. Other improvements of the expressions for the reaction rates include the running of the strong coupling constant. As shown in Ref. [25], equilibration is faster when the scale of the running coupling constant is allowed to vary with the temperature $T$.

\section{Acknowledgements}

We thank S. Bass, J. Cleymans, A. Dumitru, K. Eskola, K. Kajantie, B. Müller, M. Mustafa, J. Rafelski, D. Srivastava, and R. Venugopalan for valuable discussions. D.H.R. thanks RIKEN, Brookhaven National Laboratory, and the U.S. Department of Energy for providing the facilities essential for the completion of this work.

\section{References}

[1] see, for instance: Proc. of the XIV Int. Conf. on Ultrarelativistic Nucleus-Nucleus Collisions, "Quark Matter 99", Torino, Italy, May 10 - 15, 1999.

[2] see, for instance: E. Laermann, Nucl. Phys. A 610, 1c (1996).

[3] K.J. Eskola, K. Kajantie and J. Lindfors, Nucl. Phys. B 323, 37 (1989).

[4] K.J. Eskola and K. Kajantie, Z. Phys. C 75, 515 (1997).

[5] see, for instance: S.A. Bass, M. Gyulassy, H. Stöcker and W. Greiner, J. Phys. G 25, R1 (1999).

[6] T. Biró, E. van Doorn, B. Müller, M. Thoma and X.-N. Wang, Phys. Rev. C 48, 1275 (1993).

[7] D.K. Srivastava, M.G. Mustafa and B. Müller, Phys. Rev. C 56, 1064 (1997).

[8] B. Müller, M.G. Mustafa and D.K. Srivastava, Heavy Ion Physics 5, 387 (1997).

[9] Z. Chen, in: RHIC Summer Study '96 (Report No. BNL-52514, Brookhaven National Laboratory, 1996), 309; T. Alber et al., NA35 Collaboration, Z. Phys. C 64, 195 (1994); Eur. Phys. J. C 2, 643 (1998); I. Bearden et al., NA44 Collaboration, Phys. Lett. B 388, 431 (1996); K. Wolf et al., NA44 Collaboration, Phys. Rev. C 57, 837 (1998); H. Appelshauser et al., NA49 Collaboration, Nucl. Phys. A 638, 91 (1998).

[10] G. Rossi and G. Veneziano, Nucl. Phys. B 123, 507 (1977); Phys. Rep. 63, 153 (1980); A. Bialas, M. Bleszyń and W. Czyż, Nucl. Phys. B 111, 461 (1976); D. Kharzeev, Nucl. Phys. A 610, 418c (1996); Phys. Lett. B 378, 238 (1996); S.E. Vance, M. Gyulassy and X.-N. Wang, Nucl. Phys. A 638, 395c (1998).

[11] P. Koch, B. Müller, and J. Rafelski, Phys. Rep. 142, 167 (1986). 
[12] D.M. Elliott and D.H. Rischke, work in progress.

[13] L. Xiong and E. Shuryak, Phys. Rev. C 49, 2203 (1994).

[14] H. Heiselberg and X.-N. Wang, Phys. Rev. C 53, 1892 (1996).

[15] We thank J. Rafelski and R. Venugopalan for discussion on this point.

[16] D. Rischke, in Hadrons in Dense Matter and Hadrosynthesis, ed. J. Cleymans (Springer, Berlin, 1999), p. 21ff.

[17] M. Prakash, M. Prakash, R. Venugopalan, and G. Welke, Phys. Rept. 227, 321 (1993).

[18] J.D. Bjorken, Phys. Rev. D 27, 140 (1983).

[19] D.H. Rischke, S. Bernard and J.A. Maruhn, Nucl. Phys. A 595, 346 (1995).

[20] D.H. Rischke and M. Gyulassy, Nucl. Phys. A 608, 479 (1996).

[21] H. von Gersdorff, L. McLerran, M. Kataja, P.V. Ruuskanen, Phys. Rev. D 34, 794 (1986).

[22] K. Kajantie, hep-ph/9907544.

[23] A. Krasnitz and R. Venugopalan, hep-ph/9909203

[24] H.W. Barz, B.L. Friman, J. Knoll and H. Schulz, Nucl. Phys. A 484, 661 (1988); A 519, 831 (1990).

[25] S.M.H. Wong, Nucl. Phys. A 638, 527c (1998); Phys. Rev. C 58, 2358 (1998). 